\title{
DETECTION OF CONDUCTIVITY INCLUSIONS IN A SEMILINEAR ELLIPTIC PROBLEM ARISING FROM CARDIAC ELECTROPHYSIOLOGY
}

\author{
ELENA BERETTA * LUCA RATTI * MARCO VERANI *
}

\begin{abstract}
In this work we tackle the reconstruction of discontinuous coefficients in a semilinear elliptic equation from the knowledge of the solution on the boundary of the planar bounded domain. The problem motivated by an application in cardiac electrophysiology.

We formulate a constraint minimization problem involving a quadratic mismatch functional enhanced with a regularization term which penalizes the perimeter of the inclusion to be identified. We introduce a phase-field relaxation of the problem, employing a Ginzburg-Landau-type energy and assessing the $\Gamma$-convergence of the relaxed functional to the original one. After computing the optimality conditions of the phase-field optimization problem and introducing a discrete Finite Element formulation, we propose an iterative algorithm and prove convergence properties. Several numerical results are reported, assessing the effectiveness and the robustness of the algorithm in identifying arbitrarily-shaped inclusions.

Finally, we compare our approach to a shape derivative based technique, both from a theoretical point of view (computing the sharp interface limit of the optimality conditions) and from a numerical one.
\end{abstract}

Keywords. inverse problem; semilinear elliptic equation; phase field relaxation

AMS subject classifications. 65N21; 35J61; 35R30

1. Introduction We consider the following Neumann problem, defined over $\Omega \subset \mathbb{R}^{2}:$

$$
\left\{\begin{aligned}
-\operatorname{div}(\tilde{k}(x) \nabla y)+\chi_{\Omega \backslash \omega} y^{3}=f & \text { in } \Omega \\
\partial_{\nu} y=0 & \text { on } \partial \Omega,
\end{aligned}\right.
$$

where $\chi_{\Omega \backslash \omega}$ is the indicator function of $\Omega \backslash \omega$ and

$$
\tilde{k}(x)=\left\{\begin{array}{l}
k \text { if } x \in \omega \\
1 \text { if } x \in \Omega \backslash \omega,
\end{array}\right.
$$

being $0<k \ll 1$ and $f \in L^{2}(\Omega)$.

The boundary value problem (1.1) consists of a semilinear diffusion-reaction equation with discontinuous coefficients across the interface of an inclusion $\omega \subset \Omega$, in which the conducting properties are different from the background medium. Supposing the value of $k$ to be known, our goal is the determination of the inclusion from the knowledge of the value of $y$ on the boundary $\partial \Omega$. More precisely, given the measured data $y_{\text {meas }}$ on the boundary, we search for the inclusion $\omega \subset \Omega$ that is associated to those exact measurements, i.e. such that the corresponding solution $y$ of (1.1) satisfies

$$
\left.y\right|_{\partial \Omega}=y_{\text {meas }} .
$$

*Dipartimento di Matematica, Politecnico di Milano, P.za Leonardo da Vinci 32, 20133 Milano, Italy, \{elena.beretta,luca.ratti,marco.verani\}@polimi.it 
Since, at our knowledge, few works deal with inverse boundary value problems for nonlinear equations, the reconstruction problem analyzed in this paper is interesting from both an analytic and a numerical standpoint.

The direct problem can be related to a meaningful application arising in cardiac electrophysiology: in that context (see [39], [19]), the solution $y$ represents the electric transmembrane potential in the heart tissue, the coefficient $\tilde{k}$ is the tissue conductivity and the nonlinear reaction term encodes a ionic transmembrane current. An inclusion $\omega$ models the presence of an ischemia, which modifies substantially the conductivity properties of the tissue. The objective of our work, in the long run, is the identification of ischemic regions through a set of measurements of the electric potential acquired on the surface of the myocardium. We remark that our model is a simplified version of the more complex monodomain model (see e.g. [40], [39]). The monodomain is a continuum model which describes the evolution of the transmembrane potential on the heart tissue according to the conservation law for currents and to a satisfying description of the ionic current, which entails the coupling with a system of ordinary differential equations for the concentration of chemical species. In this preliminary setting, we remove the coupling with the ionic model, adopting instead a phenomenological description of the ionic current, through the introduction of a cubic reaction term. Moreover, we consider the stationary case in presence of a source term which plays the role of the electrical stimulus. Despite the simplifications, the problem we consider in this paper is a mathematical challenge itself. Indeed, here the difficulties include the nonlinearity of both the direct and the inverse problem, as well as the lack of measurements at disposal.

The linear counterpart of the problem, obtained when the nonlinear reaction term is removed, is strictly related to the inverse conductivity problem, also called Calderón problem, which has been object of several studies in the last decades. The problem is severly ill posed and highly nonlinear. Moreover, infinitely measurements are needed to recover smooth inclusions (see [30] and references therein). A finite number of measurements is sufficient to determine uniquely and in a stable (Lipschitz) way the inclusion only introducing additional information either on the shape of the inclusion or on its size.

Several reconstruction algorithms have been developed for the solution of the inverse conductivity problem, and it is beyond the purposes of this introduction to provide an exhaustive overview on the topic. Under the assumption that the inclusion to be reconstructed is of small size, an extended review of methods is presented in [4], many of which heavily rely on the linearity of the direct problem. Some efficient and more versatile algorithms can be derived by a variational approach, i.e., by the constraint minimization of a quadratic misfit functional, as in [31], and [3]. When dealing with the reconstruction of arbitrary inclusions in the linear case, several variational algorithms are available. A shape-optimization approach, with suitable regularization, is explored in [32] [28], [1] and [2]; in [29] this approach is coupled with topology optimization; whereas the level set technique has been applied in [38] and in [13]. Recently, 
several specific schemes have been employed to deal with the minimization of misfit functional endowed with a Total-Variation regularization: along this line we mention the Levenberg-Marquardt and Landweber algorithms in [5], the augmented Lagrangian approach in [17] and the regularized level set technique in [15]. Finally, the phase field approach has been explored for the linear inverse conductivity problem e.g in [37] and recently in [20].

Concerning inverse problems related to nonlinear PDEs, only few theoretical results and numerical strategies are available, expecially regarding the electrophysiological problem of interest. We remark that the level-set method has been implemented for the reconstruction of extended inclusions in the nonlinear problem of cardiac electrophysiology (see [34] and [16]), by evaluating the sensitivity of the cost functional with respect to a selected set of parameters involved in the full discretization of the shape of the inclusion. In [10] the authors, taking advantage from the results obtained in [8], proposed a reconstruction algorithm for the nonlinear problem (1.1) based on topological optimization, where a suitable quadratic functional is minimized to detect the position of small inclusions separated from the boundary. In [7] the results obtained in [10] and [8] have been extended to the time-dependent monodomain equation under the same assumptions.

In this paper we propose a reconstruction algorithm of inclusions of arbitrary shape and position by relying on the minimization of a suitable functional, enhanced with a perimeter penalization term, and by following a relaxation strategy relying on the phase field approach. The outline of the paper is as follows: in Section 2 we introduce the optimization problem together with its phase-field regularization, discussing wellposedness, $\Gamma$-convergence of the relaxed functional to the original one, and the derivation of necessary optimality conditions associated to the phase-field minimization problem. In Section 3 we propose an iterative reconstruction algorithm allowing for the numerical approximation of the solution and prove its convergence properties. The power of this approach is twofold: on the one hand it allows to consider conductivity inclusions of arbitrary shape and position which is the case of interest for our application and on the other it leads to good reconstructions as shown in the numerical experiments in Section 4. Finally, in Section 5 we compare our technique to a shape optimization based approach: after showing the optimality conditions derived for the relaxed problem converge to the ones corresponding to the sharp interface one, we show numerical results obtained by applying both the algorithms on the same benchmark cases.

2. Minimization problem In this section, we give a rigorous formulation both of the direct and of the inverse problem under investigation. The analysis of the wellposedness of the direct problem is reported in details, and consists in an extension of the results previously obtained in [8]. The inverse problem is instead associated to a constraint minimization problem for which we introduce a regularization and relaxation strategy in order to overcome instability and to allow the implementation of a reconstruction algorithm. 
We formulate the problems (1.1) and (1.2) in terms of the indicator function of the inclusion, $u=\chi_{\omega}$. We assume an a priori hypothesis on the inclusion, namely that it is a subset of $\Omega$ of finite perimeter: $u$ belongs to $B V(\Omega)=\left\{v \in L^{1}(\Omega): T V(v)<\infty\right\}$, being

$$
T V(v)=\sup \left\{\int_{\Omega} v \operatorname{div}(\phi) ; \quad \phi \in C_{0}^{1}\left(\Omega ; \mathbb{R}^{2}\right),\|\phi\|_{L^{\infty}} \leq 1\right\}
$$

endowed with the norm $\|\cdot\|_{B V}=\|\cdot\|_{L^{1}}+T V(\cdot)$. Moreover, we formulate particular restrictions on the inclusion and on the source $f$.

Assumption 2.1. Given a positive number $d_{0}$ we assume that

$$
u \in X_{0,1}=\left\{v \in B V(\Omega): v(x) \in\{0,1\} \text { a.e. in } \Omega, u=0 \text { a.e. in } \Omega^{d_{0}}\right\} \text {, }
$$

where $\Omega^{d_{0}}=\left\{x\right.$ s.t. $\left.\operatorname{dist}(x, \partial \Omega) \leq d_{0}\right\}$. This also entails that the inclusion is well separated from the boundary $\partial \Omega$. Moreover,

Assumption 2.2. Given a positive constant $m$, we require

$$
f \geq m \text { a.e. in } \Omega \text {. }
$$

The weak formulation of the direct problem (1.1) in terms of $u$ reads: find $y$ in $H^{1}(\Omega)$ s.t., $\forall \varphi \in H^{1}(\Omega)$,

$$
\int_{\Omega} a(u) \nabla y \nabla \varphi+\int_{\Omega} b(u) y^{3} \varphi=\int_{\Omega} f \varphi,
$$

being $a(u)=1-(1-k) u$ and $b(u)=1-u$. Define $S: X_{0,1} \rightarrow H^{1}(\Omega)$ the solution map: for all $u \in X_{0,1}, S(u)=y$ is the solution to problem (2.3) with indicator function $u$; the inverse problem consists in:

$$
\text { find } u \in X_{0,1} \text { s.t. }\left.S(u)\right|_{\partial \Omega}=y_{\text {meas }} \text {. }
$$

In the proof of various proposition we have make use of the following generalized Poincaré inequality:

Lemma 2.1. $\exists C>0, C=C\left(\Omega, d_{0}\right)$ s.t., $\forall w \in H^{1}(\Omega)$,

$$
\|w\|_{H^{1}(\Omega)}^{2} \leq C\left(\|\nabla w\|_{L^{2}(\Omega)}^{2}+\|w\|_{L^{2}\left(\Omega^{d_{0}}\right)}^{2}\right) .
$$

The proof of the Lemma 2.1 is given in the Appendix of [8] and easily follows by Theorem 8.11 in [33].

Thanks to Lemma 2.1, we can prove the following well-posedness result for the direct problem.

Proposition 2.1. Consider $f \in\left(H^{1}(\Omega)\right)^{*}$ and a function $u \in X_{0,1}$. Then there exists an unique solution $S(u) \in H^{1}(\Omega)$ of

$$
\int_{\Omega} a(u) \nabla S(u) \cdot \nabla v+\int_{\Omega} b(u) S(u)^{3} v=\int_{\Omega} f v \quad \forall v \in H^{1}(\Omega),
$$

where $a(u)=1-(1-k) u$ and $b(u)=1-u$. 
Proof. The proof is analogous to the analysis performed in [8, Theorem 4.1], but generalises that result to the case of inclusions of finite perimeter. The strategy consists in applying the Minty-Browder theorem on the direct operator $T: H^{1}(\Omega) \rightarrow\left(H^{1}(\Omega)\right)^{*}$ s.t.

$$
\langle T(S), v\rangle_{*}=\int_{\Omega} a(u) \nabla S \cdot \nabla v+\int_{\Omega} b(u) S^{3} v
$$

which shows to be continuous, coercive and strictly monotone. In particular

- Local Lipschitz continuity:

$$
\begin{aligned}
\left|\left\langle T(S)-T\left(S_{0}\right), v\right\rangle_{*}\right| & =\left|\int_{\Omega} a(u) \nabla\left(S-S_{0}\right) \cdot \nabla v+\int_{\Omega} b(u)\left(S-S_{0}\right) q v\right| \\
& \leq\left\|\nabla\left(S-S_{0}\right)\right\|_{L^{2}}\|\nabla v\|_{L^{2}}+\left\|S-S_{0}\right\|_{L^{6}}\|q\|_{L^{3}}\|v\|_{L^{2}},
\end{aligned}
$$

(being $q=S^{2}+S S_{0}+S_{0}^{2}$ ). If $S$ and $S_{0}$ belong to a bounded subset of $H^{1}(\Omega)$, then (thanks to the Sobolev Embedding of $H^{1}(\Omega)$ in $L^{6}(\Omega)$ ) we can assess that $\|q\|_{L^{3}} \leq M$ and moreover $\exists K=K(u)>0$ s.t.

$$
\left|\left\langle T(S)-T\left(S_{0}\right), v\right\rangle_{*}\right| \leq K\left\|S-S_{0}\right\|_{H^{1}}\|v\|_{H^{1}} \quad \forall v \in H^{1}(\Omega) .
$$

- Coercivity: we show that $\langle T(S), S\rangle_{*} \rightarrow+\infty$ as $\|S\|_{H^{1}(\Omega)} \rightarrow+\infty$. Since $u=0$ a.e. in $\Omega^{d_{0}}, b(u) \geq \chi_{\Omega^{d_{0}}}$, the indicator function of $\Omega^{d_{0}}$. Then,

$$
\begin{aligned}
\langle T(S), S\rangle_{*} & \geq k \int_{\Omega}|\nabla S|^{2}+\int_{\Omega^{d_{0}}} S^{4} \geq k\|\nabla S\|_{L^{2}(\Omega)}^{2}+\frac{1}{|\Omega|}\|S\|_{L^{2}\left(\Omega^{d_{0}}\right)}^{4} \\
& =k\left(\|\nabla S\|_{L^{2}(\Omega)}^{2}+\|S\|_{L^{2}\left(\Omega^{d_{0}}\right)}^{2}\right)+R
\end{aligned}
$$

where $R=\frac{1}{|\Omega|}\|S\|_{L^{2}\left(\Omega^{d_{0}}\right)}^{4}-k\|S\|_{L^{2}\left(\Omega^{d_{0}}\right)}^{2}$ can be bounded by below independently of $S: R \geq-\frac{k^{2}|\Omega|}{4}$. Together with Poincaré inequality in Lemma 2.1, we conclude that

$$
\langle T(S), S\rangle_{*} \geq \frac{k}{C}\|S\|_{H^{1}(\Omega)}^{2}-\frac{k^{2}|\Omega|}{4} .
$$

- (Strict) monotonicity: we claim that $\langle T(S)-T(R), S-R\rangle_{*} \geq 0$ and $\langle T(S)-$ $T(R), S-R\rangle_{*}=0 \Leftrightarrow S=R$. Indeed,

$$
\langle T(S)-T(R), S-R\rangle_{*} \geq \int_{\Omega} k|\nabla(S-R)|^{2}+\int_{\Omega^{d_{0}}}\left(S^{2}+S R+R^{2}\right)(S-R)^{2} \geq 0 .
$$

Moreover, since $S^{2}+S R+R^{2} \geq \frac{1}{4}(S-R)^{2}$,

$$
\langle T(S)-T(R), S-R\rangle_{*}=0 \Rightarrow\|\nabla(S-R)\|_{L^{2}(\Omega)}=0 \text { and } \int_{\Omega^{d_{0}}}(S-R)^{4}=0
$$

and from the latter equality it follows that $S=R$ a.e. in $\Omega^{d_{0}}$, hence also $\|S-R\|_{L^{2}\left(\Omega^{\left.d_{0}\right)}\right.}=0$, and via Lemma $2.1\|S-R\|_{H^{1}(\Omega)}=0$. 
It is possible to prove additional properties of the solution $S(u)$ of the direct problem. In particular, we provide an uniform bound on $\|S(u)\|_{H^{1}(\Omega)}$ independent of $u$. Proposition 2.2. There exists a constant $C=C\left(\Omega, d_{0}, k\right)$ s.t., $\forall u \in X_{0,1}$,

$$
\|S(u)\|_{H^{1}(\Omega)} \leq C\left(\|f\|_{L^{2}(\Omega)}+\|f\|_{L^{2}(\Omega)}^{3}\right) .
$$

This can be proved as in [8, Proposition 4.1], where we take advantage of the bound

$$
\|S(u)\|_{L^{2}\left(\Omega^{d_{0}}\right)}^{4} \leq\left|\Omega^{d_{0}}\right| \int_{\Omega^{d_{0}}} S(u)^{4} \leq|\Omega| \int_{\Omega} b(u) S(u)^{4},
$$

and hence the constant appearing in (2.6) only depends on $\Omega, d_{0}, k$.

Moreover, we prove a Hölder regularity result on $S(u)$ :

Proposition 2.3. Let $S(u)$ be the solution of (2.3) associated to $u \in X_{0,1}$ and let $f \in L^{2}(\Omega)$. Then, $S(u) \in C^{\alpha}(\bar{\Omega})$ and

$$
\|S(u)\|_{C^{\alpha}(\bar{\Omega})} \leq C\left(\Omega, k,\|f\|_{L^{2}(\Omega)}, d_{0}\right)
$$

Proof. The proof is analogous to the one in [8]. An application of [26, Theorem 8.24] ensures that

$$
\forall \Omega^{\prime} \subset \subset \Omega, \quad\|S(u)\|_{C^{\alpha}\left(\overline{\Omega^{\prime}}\right)} \leq C\left(\|S(u)\|_{L^{2}(\Omega)}+\|S(u)\|_{L^{6}(\Omega)}^{3}+\|f\|_{L^{2}(\Omega)}\right) \leq C,
$$

where $C=C\left(\overline{\Omega^{\prime}}, k,\|f\|_{L^{2}(\Omega)}\right)$. By taking $\Omega^{\prime} \supset \Omega^{d_{0}}$, since the conductivity is constant in $\Omega_{d_{0}}$ and the normal derivative on the boundary is zero, we can apply standard regularity results up to the boundary, obtaining:

$$
\|S(u)\|_{C^{\alpha}(\bar{\Omega})} \leq C=C\left(\Omega, d_{0}, k,\|f\|_{L^{2}(\Omega)}\right) .
$$

Finally, we prove an estimate which occurs many times in the proof of various results.

Proposition 2.4. Suppose that $f \in L^{2}(\Omega)$ s.t. $f \geq m>0$ a.e. in $\Omega$. Consider $S(u)$ the solution of problem (2.3) associated to $u \in X_{0,1}$. Then, $S(u) \geq m^{1 / 3}$.

The proof is an immediate consequence of the following Lemma:

LEMMA 2.2. Let $S_{1}$ and $S_{2}$ be a sub- and supersolution of (2.3) with $u \in X_{0,1}$, namely $S_{1}, S_{2} \in H^{1}(\Omega)$ s.t., $\forall \varphi \in H^{1}(\Omega), \varphi \geq 0$ a.e., it holds:

$$
\begin{aligned}
& \int_{\Omega} a(u) \nabla S_{1} \cdot \nabla \varphi+\int_{\Omega} b(u) S_{1}^{3} \varphi-\int_{\Omega} f \varphi \leq 0, \\
& \int_{\Omega} a(u) \nabla S_{2} \cdot \nabla \varphi+\int_{\Omega} b(u) S_{2}^{3} \varphi-\int_{\Omega} f \varphi \geq 0 .
\end{aligned}
$$

Then, $S_{1} \leq S_{2}$ a.e. in $\Omega$. 
Proof. Subtract the equations (2.8) - (2.7) and define $W=S_{2}-S_{1}$ : it holds, $\forall \varphi \in$ $H^{1}(\Omega), \varphi \geq 0$ a.e.,

$$
\int_{\Omega} a(u) \nabla W \cdot \nabla \varphi+\int_{\Omega} b(u) Q W \varphi \geq 0
$$

where $Q=\left(S_{1}^{2}+S_{1} S_{2}+S_{2}^{2}\right) \geq 0$. Take $\varphi=W^{-}$, the negative part of $W$. We remark that $W^{+}=\max \{0, W\}, W^{-}=\max \{0,-W\}, W=W^{+}-W^{-} ;$moreover $W^{+}, W^{-} \in H^{1}(\Omega)$, $W^{+} W^{-}=0$, and in view of [24, Theorem 4.4] we refer to $\nabla W^{-}$as the gradient of the negative part $W^{-}$or equivalently as the vector of the negative parts of the components of $\nabla W$. Thus, it holds

$$
\int_{\Omega} a(u) \nabla W^{-} \cdot \nabla W^{-}+\int_{\Omega} b(u) Q\left(W^{-}\right)^{2} \leq 0
$$

which implies that $S_{2} \geq S_{1}$ a.e. Indeed, $k\left\|\nabla W^{-}\right\|_{L^{2}(\Omega)} \leq 0$ implies $\nabla W^{-}=0$ a.e. in $\Omega$; moreover, both $S_{1}$ and $S_{2}$ are continuous, and hence also $W$ and $W^{-}$, which entails $W^{-}=c, c \geq 0$ by definition. In order to guarantee that $W^{-}=\max \{0,-W\}=c$ is continuous, either $c=0$ or $W=-c<0$ in $\Omega$. The latter case, though, would imply that $S_{2}=S_{1}-c$ and, by simple computation, $Q=3 S_{1}^{2}-3 c S_{1}+c^{2} \geq \frac{c^{2}}{4}$, which is incompatible with $\int_{\Omega} b(u) Q\left(W^{-}\right)^{2} \leq 0$. Hence $W^{-}=0$, and so $W=W^{+} \geq 0$.

Proof. [Proof of Proposition 2.4] Taking $S_{2}=S(u)$ and $S_{1}=m^{1 / 3}$ (which is a subsolution since $b(u) m-f \leq 0)$, we obtain the uniform bound $S(u) \geq m^{1 / 3}$.

REMARK 2.1. We could extend all the previous results to a class of more general functions $f$, namely $f$ not vanishing in $\Omega^{d_{0}}$, but that would entail that the lower bound in Proposition 2.4 might depend on $u$. On the other hand, when applying the previous estimates in the proofs of following results (in particular, Proposition 2.5, 2.10, 5.1 and Lemma 3.1), we always invoke Proposition 2.4 on a fixed indicator function $u$.

The crucial property satisfied by the solution map is the continuity with respect to the $L^{1}$ norm, which requires an accurate treatment due to the nonlinearity of the direct problem.

Proposition 2.5. Let $f \in L^{2}(\Omega)$ satisfy assumption (2.2). If $\left\{u_{n}\right\} \subset X_{0,1}$ s.t. $u_{n} \stackrel{L^{1}}{\longrightarrow}$ $u \in X_{0,1}$, then $\left.\left.S\left(u_{n}\right)\right|_{\partial \Omega} \stackrel{L^{2}(\partial \Omega)}{\longrightarrow} S(u)\right|_{\partial \Omega}$.

Proof. Define $w_{n}=S\left(u_{n}\right)-S(u)$; then, subtracting (2.3) evaluated at $u_{n}$ and the same one evaluated at $u, w_{n}$ is the solution of:

$$
\int_{\Omega} a\left(u_{n}\right) \nabla w_{n} \nabla \varphi+\int_{\Omega} b\left(u_{n}\right) q_{n} w_{n} \varphi=\int_{\Omega}(1-k)\left(u_{n}-u\right) \nabla S(u) \nabla \varphi-\int_{\Omega}\left(u_{n}-u\right) S(u)^{3} \varphi,
$$

where $q_{n}=S\left(u_{n}\right)^{2}+S\left(u_{n}\right) S(u)+S(u)^{2}$. Considering $\varphi=w_{n}$ and taking advantage of the fact that $a\left(u_{n}\right) \geq k$ and (by simple computation) $q_{n} \geq \frac{3}{4} S(u)^{2}$, we can show, via 
Cauchy-Schwarz inequality, that

$$
\begin{aligned}
k\left\|\nabla w_{n}\right\|_{L^{2}(\Omega)}^{2}+\frac{3}{4} \int_{\Omega} b\left(u_{n}\right) S(u)^{2} w_{n}^{2} \leq & (1-k)\left\|\left(u_{n}-u\right) \nabla S(u)\right\|_{L^{2}(\Omega)}\left\|\nabla w_{n}\right\|_{L^{2}(\Omega)} \\
& +\left\|\left(u_{n}-u\right) S(u)^{3}\right\|_{L^{2}(\Omega)}\left\|w_{n}\right\|_{L^{2}(\Omega)} .
\end{aligned}
$$

We remark that $\left(u_{n}-u\right) S(u)^{3} \in L^{2}(\Omega)$ since $S(u) \in H^{1}(\Omega) \subset \subset L^{6}(\Omega)$. Moreover, as $b\left(u_{n}\right) \geq \chi_{\Omega^{d_{0}}}$ and using Proposition 2.4,

$$
\begin{aligned}
k\left\|\nabla w_{n}\right\|_{L^{2}(\Omega)}^{2}+\frac{3}{4} \int_{\Omega^{d_{0}}} m^{2 / 3} w_{n}^{2} \leq & (1-k)\left\|\left(u_{n}-u\right) \nabla S(u)\right\|_{L^{2}(\Omega)}\left\|\nabla w_{n}\right\|_{L^{2}(\Omega)} \\
& +\left\|\left(u_{n}-u\right) S(u)^{3}\right\|_{L^{2}(\Omega)}\left\|w_{n}\right\|_{L^{2}(\Omega)},
\end{aligned}
$$

from which we deduce

$$
k\left\|\nabla w_{n}\right\|_{L^{2}(\Omega)}^{2}+\frac{3}{4} m^{2 / 3}\left\|w_{n}\right\|_{L^{2}\left(\Omega^{\left.d_{0}\right)}\right.}^{2} \leq\left(q_{1}+q_{2}\right)\left\|w_{n}\right\|_{H^{1}(\Omega)},
$$

where $q_{1}=\left\|\left(u_{n}-u\right) \nabla S(u)\right\|_{L^{2}(\Omega)}$ and $q_{2}=\left\|\left(u_{n}-u\right) S(u)^{3}\right\|_{L^{2}(\Omega)}$, which implies, thanks to the Poincaré inequality in Lemma 2.1,

$$
\left\|w_{n}\right\|_{H^{1}(\Omega)} \leq C\left(q_{1}+q_{2}\right)
$$

being $C=C\left(d_{0}, \Omega, m, k\right)$. Consider

$$
q_{1}=\left(\int_{\Omega}\left(u_{n}-u\right)^{2}|\nabla S(u)|^{2}\right)^{\frac{1}{2}}
$$

since $u_{n} \stackrel{L^{1}}{\longrightarrow} u$, then (up to a subsequence) $u_{n} \rightarrow u$ pointwise almost everywhere. Thus also the integrand $\left(u_{n}-u\right)^{2}|\nabla S(u)|^{2}$ converges to 0 . Moreover, $\left|u_{n}-u\right| \leq 1$, hence $\forall n$ $\left(u_{n}-u\right)^{2}|\nabla S(u)|^{2} \leq|\nabla S(u)|^{2} \in L^{1}(\Omega)$, and thanks to Lebesgue convergence theorem, we conclude that $q_{1} \rightarrow 0$. Analogously, $q_{2} \rightarrow 0$ and eventually $\left\|w_{n}\right\|_{H^{1}(\Omega)} \rightarrow 0$, i.e. $S\left(u_{n}\right) \stackrel{H^{1}}{\longrightarrow}$ $S(u)$ and by the trace inequality also $\left.\left.S\left(u_{n}\right)\right|_{\partial \Omega} \stackrel{L^{2}(\partial \Omega)}{\longrightarrow} S(u)\right|_{\partial \Omega}$.

Remark 2.2. Being $X_{0,1}$ a closed subspace of the Banach space $B V(\Omega)$, it is compact with respect to its weak topology; moreover, the weak BV convergence implies the strong $L^{1}$ convergence, and in view of Proposition 2.5 we can assess that the map $F=\tau \circ S$, $\tau$ being the trace operator in $H^{1}(\Omega)$, is compact from $X_{0,1}$ to $L^{2}(\partial \Omega)$. It is immediate to conclude that, if the inverse $F^{-1}$ exists, it cannot be continuous: hence, the inverse problem (2.4) is ill-posed.

We now introduce the following constraint optimization problem:

$$
\underset{u \in X_{0,1}}{\operatorname{argmin}} J(u) ; \quad J(u)=\frac{1}{2}\left\|S(u)-y_{\text {meas }}\right\|_{L^{2}(\partial \Omega)}^{2},
$$

which shares the same property of non-stability and (possibly) non-uniqueness as problem (2.4). Nevertheless, a well-known strategy to recover well-posedness for problem 
(2.10) is to introduce a Tikhonov regularization term in the functional to minimize, e.g. a penalization term for the perimeter of the inclusion. The regularized problem reads:

$$
\underset{u \in X_{0,1}}{\operatorname{argmin}} J_{r e g}(u) ; \quad J_{r e g}(u)=\frac{1}{2}\left\|S(u)-y_{\text {meas }}\right\|_{L^{2}(\partial \Omega)}^{2}+\alpha T V(u),
$$

For the regularized problem (2.11), it is possible to prove several desirable properties:

- for every $\alpha>0$ there exists at least one solution to (2.11) (existence);

- small perturbations on the data $y_{\text {meas }}$ in $L^{2}(\partial \Omega)$-norm imply small perturbation on the solutions of (2.11) in $B V$-intermediate convergence (stability);

- the sequence of solutions of problem (2.11) associated to the regularization parameters $\left\{\alpha_{k}\right\}$ (s.t. $\alpha_{k} \rightarrow 0$ ) converges in the $B V$-intermediate convergence to a minimum-variation solution of problem (2.10).

We point out that a sequence $\left\{u_{n}\right\} \subset B V(\Omega)$ converges to $u \in B V(\Omega)$ in the sense of the intermediate convergence iff $u_{n} \stackrel{L^{1}}{\rightarrow} u$ and $T V\left(u_{n}\right) \rightarrow T V(u)$. The proof of the previous properties follows from a careful application of the results in [23, Chapter 10], taking into account that $B V(\Omega)$ is a non-reflexive Banach space.

According to the stated results, a good approximation of a minimum-variation solution of the inverse problem (2.4) can be achieved by solving the regularized constraint minimization problem (2.11) with a sufficiently small parameter $\alpha>0$. Although the stability of the problem is guaranteed, its numerical solution may raise difficulties, namely the non-convexity both of the functional $J_{\text {reg }}$ and of the space $X_{0,1}$, as well as the non-differentiability of the functional. To overcome these difficulties, in this work we propose a phase-field relaxation of the optimization problem (2.11) inspired by [20], with the additional difficulty of the nonlinearity of the direct problem. The relaxation strategy consists in defining a minimization problem in a space of more regular functions, associated to a differentiable cost functional (which in our case is achieved by replacing the Total Variation term with a Modica-Mortola functional, representing a Ginzburg-Landau energy).

Consider $u \in \mathcal{K}=\left\{v \in H^{1}(\Omega): 0 \leq v \leq 1\right.$ a.e. in $\Omega, v=0$ a.e. in $\left.\Omega^{d_{0}}\right\}$ and, for every $\varepsilon>0$, introduce the optimization problem:

$$
\underset{u \in \mathcal{K}}{\arg \min } J_{\varepsilon}(u) ; \quad J_{\varepsilon}(u)=\frac{1}{2}\left\|S(u)-y_{\text {meas }}\right\|_{L^{2}(\partial \Omega)}^{2}+\alpha \int_{\Omega}\left(\varepsilon|\nabla u|^{2}+\frac{1}{\varepsilon} u(1-u)\right) .
$$

The well-posedness result for the direct problem in Proposition 2.1, together with the additional stability and regularity results can be easily extended to the case $u \in \mathcal{K}$. In the next two propositions, we prove existence and stability of the solutions of the relaxed minimization problem (2.12) for fixed $\varepsilon$.

Proposition 2.6. For every fixed $\varepsilon>0$, the minimization problem (2.12) has a solution $u_{\varepsilon} \in \mathcal{K}$.

Proof. Fix $\varepsilon>0$ and consider a minimizing sequence for the functional $J_{\varepsilon},\left\{u_{k}\right\} \subset \mathcal{K}$ (we omit the dependence of $u_{k}$ on $\varepsilon$ ). By definition of minimizing sequence, $J_{\varepsilon}\left(u_{k}\right) \leq M$ independently of $k$, which implies that also $\left\|\nabla u_{k}\right\|_{L^{2}(\Omega)}^{2}$ is bounded. Moreover, being 
$u_{k} \in \mathcal{K}, 0 \leq u_{k} \leq 1$ a.e., thus $\left\|u_{k}\right\|_{L^{2}(\Omega)}$ and $\left\|u_{k}\right\|_{H^{1}(\Omega)}$ are bounded independently of $k$. Thanks to weak compactness of $H^{1}$, there exist $u_{\varepsilon} \in H^{1}(\Omega)$ and a subsequence $\left\{u_{k_{n}}\right\}$ s.t. $u_{k_{n}} \stackrel{H^{1}}{\longrightarrow} u_{\varepsilon}$, hence $u_{k_{n}} \stackrel{L^{2}}{\rightarrow} u_{\varepsilon}$. The strong $L^{2}$ convergence implies (up to a subsequence) pointwise convergence a.e., which allows to conclude (together with the Lebesgue's dominated convergence theorem, since $\left.u_{k_{n}}\left(1-u_{k_{n}}\right) \leq 1 / 2\right)$ that

$$
\int_{\Omega} u_{k_{n}}\left(1-u_{k_{n}}\right) \rightarrow \int_{\Omega} u_{\varepsilon}\left(1-u_{\varepsilon}\right) .
$$

Moreover, by the lower semicontinuity of the $H^{1}$ norm with respect to the weak convergence, and by the compact embedding in $L^{2}$,

$$
\begin{aligned}
\left\|u_{\varepsilon}\right\|_{H^{1}(\Omega)}^{2} & \leq \liminf _{n}\left\|u_{k_{n}}\right\|_{H^{1}(\Omega)}^{2} \\
\left\|u_{\varepsilon}\right\|_{L^{2}(\Omega)}^{2}+\left\|\nabla u_{\varepsilon}\right\|_{L^{2}(\Omega)}^{2} & \leq \lim _{n}\left\|u_{k_{n}}\right\|_{L^{2}(\Omega)}^{2}+\liminf _{n}\left\|\nabla u_{k_{n}}\right\|_{L^{2}(\Omega)}^{2} \\
\left\|\nabla u_{\varepsilon}\right\|_{L^{2}(\Omega)}^{2} & \leq \liminf _{n}\left\|\nabla u_{k_{n}}\right\|_{L^{2}(\Omega)}^{2} .
\end{aligned}
$$

Moreover, using the continuity of the solution map $S$ with respect to the $L^{1}$ convergence, we can conclude that

$$
J_{\varepsilon}\left(u_{\varepsilon}\right) \leq \liminf _{n} J_{\varepsilon}\left(u_{k_{n}}\right)
$$

Finally, by pointwise convergence, $0 \leq u_{\varepsilon} \leq 1$ a.e. and $u_{\varepsilon}=0$ a.e. in $\Omega^{d_{0}}$, hence $u_{\varepsilon}$ is a minimum of $J_{\varepsilon}$ in $\mathcal{K}$.

Proposition 2.7. Fix $\alpha, \varepsilon>0$ and consider a sequence $\left\{y^{k}\right\} \subset L^{2}(\partial \Omega)$ such that $y^{k} \stackrel{L^{2}(\partial \Omega)}{\longrightarrow} y_{\text {meas }}$. For each $k$, let $u_{\varepsilon}^{k}$ be a solution of (2.12), where $y_{\text {meas }}$ is replaced by $y^{k}$. Then, up to a subsequence, $u_{\varepsilon}^{k} \stackrel{H^{1}}{\longrightarrow} u_{\varepsilon}$, where $u_{\varepsilon}$ is a solution of (2.12).

Proof. Consider a solution $u^{*}$ of (2.12): by definition of $u_{\varepsilon}^{k}$, it holds

$$
\begin{aligned}
& \frac{1}{2}\left\|S\left(u_{\varepsilon}^{k}\right)-y^{k}\right\|_{L^{2}(\partial \Omega)}^{2}+\alpha \varepsilon\left\|\nabla u_{\varepsilon}^{k}\right\|_{L^{2}(\Omega)}^{2}+\frac{\alpha}{\varepsilon} \int_{\Omega} u_{\varepsilon}^{k}\left(1-u_{\varepsilon}^{k}\right) \\
& \quad \leq \frac{1}{2}\left\|S\left(u^{*}\right)-y^{k}\right\|_{L^{2}(\partial \Omega)}^{2}+\alpha \varepsilon\left\|\nabla u^{*}\right\|_{L^{2}(\Omega)}^{2}+\frac{\alpha}{\varepsilon} \int_{\Omega} u^{*}\left(1-u^{*}\right) \\
& \quad \leq \frac{1}{2}\left\|y_{\text {meas }}-y^{k}\right\|_{L^{2}(\partial \Omega)}^{2}+\frac{1}{2} J_{\varepsilon}\left(u^{*}\right) .
\end{aligned}
$$

Hence $\left\|\nabla u_{\varepsilon}^{k}\right\|_{L^{2}(\Omega)}$ is bounded independently of $k$, and so is $\left\|u_{\varepsilon}^{k}\right\|_{L^{2}(\Omega)}$ (since $\left.u_{\varepsilon}^{k} \in \mathcal{K}\right)$. This implies that, up to a subsequence, $u_{\varepsilon}^{k} \stackrel{H^{1}}{\longrightarrow} u_{\varepsilon} \in H^{1}(\Omega)$, from which it follows that $u_{\varepsilon}^{k} \stackrel{L^{2}}{\rightarrow} u_{\varepsilon}$ and in particular $S\left(u_{\varepsilon}^{k}\right) \stackrel{H^{1}}{\longrightarrow} S\left(u_{\varepsilon}\right)$ (thanks to Proposition 2.5) and $u_{\varepsilon}^{k} \rightarrow u_{\varepsilon}$ almost everywhere in $\Omega$, and by Lebesgue's convergence theorem also $\int_{\Omega} u_{\varepsilon}^{k}\left(1-u_{\varepsilon}^{k}\right) \rightarrow$ $\int_{\Omega} u_{\varepsilon}\left(1-u_{\varepsilon}\right)$. Finally, by lower semi-continuity of the $H^{1}$ norm with respect to the weak convergence, we conclude that

$$
\begin{aligned}
J_{\varepsilon}\left(u_{\varepsilon}\right) & \leq \liminf _{k}\left(\frac{1}{2}\left\|S\left(u_{\varepsilon}^{k}\right)-y^{k}\right\|_{L^{2}(\partial \Omega)}^{2}+\alpha \varepsilon\left\|\nabla u_{\varepsilon}^{k}\right\|_{L^{2}(\Omega)}^{2}+\frac{\alpha}{\varepsilon} \int_{\Omega} u_{\varepsilon}^{k}\left(1-u_{\varepsilon}^{k}\right)\right) \\
& \leq J_{\varepsilon}\left(u^{*}\right)+\frac{1}{2} \lim _{k}\left\|y_{\text {meas }}-y^{k}\right\|_{L^{2}(\partial \Omega)}^{2},
\end{aligned}
$$


hence $J_{\varepsilon}\left(u_{\varepsilon}\right)=J_{\varepsilon}\left(u^{*}\right)$ and $u_{\varepsilon}$ is a solution of (2.12). Moreover, this implies that $\left\|\nabla u_{\varepsilon}\right\|_{L^{2}(\Omega)}=\lim _{k}\left\|\nabla u_{\varepsilon}^{k}\right\|_{L^{2}(\Omega)}$; and since $H^{1}$ is an Hilbert space, together with the weak convergence, this implies that $u_{\varepsilon}^{k} \stackrel{H^{1}}{\longrightarrow} u_{\varepsilon}$.

The asymptotic behaviour of the phase-field relaxation when $\varepsilon \rightarrow 0$ is reported in the next two propositions and is related to the notion of $\Gamma$-convergence.

Proposition 2.8. Consider the space $X$ of the Lebesgue-measurable functions over $\Omega$ endowed with the $L^{1}(\Omega)$ norm and the following extension of the cost functionals on $X$

$$
\tilde{J}=\left\{\begin{array}{rl}
J_{\text {reg }}(u) & \text { if } u \in X_{0,1} \\
\infty & \text { otherwise },
\end{array} \quad \tilde{J}_{\varepsilon}=\left\{\begin{aligned}
J_{\varepsilon}(u) & \text { if } u \in \mathcal{K} \\
\infty & \text { otherwise. }
\end{aligned}\right.\right.
$$

Then, the functionals $\tilde{J}_{\varepsilon_{k}}$ associated to $\left\{\varepsilon_{k}\right\}$ s.t. $\varepsilon_{k} \rightarrow 0$ converge to $\tilde{J}$ in $X$ in the sense of the $\Gamma$-convergence.

The proof can be obtained by adapting the one of [20, Theorem 6.1]. Moreover, from the compactness result in [6, Proposition 4.1] and by the definition of $\Gamma$-convergence, it is easy to prove the following convergence result for the solutions of (2.12).

Proposition 2.9. Consider a sequence $\left\{\varepsilon_{k}\right\}$ s.t. $\varepsilon_{k} \rightarrow 0$ and let $\left\{u_{\varepsilon_{k}}\right\}$ be the sequence of the respective minimizers of the functionals $\left\{J_{\varepsilon_{k}}\right\}$. Then, there exists a subsequence, still denoted as $\left\{\varepsilon_{k}\right\}$ and a function $u \in X_{0,1}$ such that $u_{\varepsilon_{k}} \rightarrow u$ in $L^{1}$ and $u$ is a solution of (2.11).

2.1. Optimality conditions We can now provide an expression for the optimality condition associated with the minimization problem (2.12), which is formulated as a variational inequality involving the Fréchet derivative of $J_{\varepsilon}$.

Proposition 2.10. Consider the solution map $S: \mathcal{K} \rightarrow H^{1}(\Omega)$ and let $f \in L^{2}(\Omega)$ satisfy assumption (2.2): for every $\varepsilon>0$, the operators $S$ and $J_{\varepsilon}$ are Fréchet-differentiable on $\mathcal{K} \subset L^{\infty}(\Omega) \cap H^{1}(\Omega)$ and a minimizer $u_{\varepsilon}$ of $J_{\varepsilon}$ satisfies the variational inequality:

$$
J_{\varepsilon}^{\prime}\left(u_{\varepsilon}\right)\left[v-u_{\varepsilon}\right] \geq 0 \quad \forall v \in \mathcal{K}
$$

being

$$
J_{\varepsilon}^{\prime}(u)[\vartheta]=\int_{\Omega}(1-k) \vartheta \nabla S(u) \cdot \nabla p+\int_{\Omega} \vartheta S(u)^{3} p+2 \alpha \varepsilon \int_{\Omega} \nabla u \cdot \nabla \vartheta+\frac{\alpha}{\varepsilon} \int_{\Omega}(1-2 u) \vartheta
$$

where $\vartheta \in \mathcal{K}-u=\{v$ s.t. $u+v \in \mathcal{K}\}$ and $p$ is the solution of the adjoint problem:

$$
\int_{\Omega} a(u) \nabla p \cdot \nabla \psi+\int_{\Omega} 3 b(u) S(u)^{2} p \psi=\int_{\partial \Omega}\left(S(u)-y_{\text {meas }}\right) \psi \quad \forall \psi \in H^{1}(\Omega) .
$$

Proof. First of all we need to prove that $S$ is Fréchet differentiable in $L^{\infty}(\Omega)$ : in particular, we claim that for $\vartheta \in L^{\infty}(\Omega) \cap(\mathcal{K}-u)$ it holds that $S^{\prime}(u)[\vartheta]=S_{*}$, where $S_{*}$ is the solution in $H^{1}(\Omega)$ of

$$
\int_{\Omega} a(u) \nabla S_{*} \nabla \varphi+\int_{\Omega} b(u) 3 S(u)^{2} S_{*} \varphi=\int_{\Omega}(1-k) \vartheta \nabla S \nabla \varphi+\int_{\Omega} \vartheta S(u)^{3} \varphi \quad \forall \varphi \in H^{1}(\Omega),
$$


namely, that

$$
\left\|S(u+\vartheta)-S(u)-S_{*}\right\|_{H^{1}(\Omega)}=o\left(\|\vartheta\|_{L^{\infty}(\Omega)}\right) .
$$

First we show that if $\vartheta \in L^{\infty}(\Omega) \cap(\mathcal{K}-u)$, then $\|S(u+\vartheta)-S(u)\|_{H^{1}(\Omega)} \leq C\|\vartheta\|_{L^{\infty}(\Omega)}$. Indeed, the difference $w=S(u+\vartheta)-S(u)$ satisfies

$$
\begin{aligned}
\int_{\Omega} a(u+\vartheta) \nabla w \nabla \varphi+\int_{\Omega} b(u+\vartheta) q w \varphi= & -\int_{\Omega}(a(u+\vartheta)-a(u)) \nabla S(u) \nabla \varphi \\
& -\int_{\Omega}(b(u+\vartheta)-b(u)) S(u)^{3} \varphi \quad \forall \varphi \in H^{1}(\Omega),
\end{aligned}
$$

with $q=S(u+\vartheta)^{2}+S(u) S(u+\vartheta)+S(u)^{2}$. Substituting $a(u+\vartheta)-a(u)=-(1-k) \vartheta$ and $b(u+\vartheta)-b(u)=-\vartheta$, and taking $\varphi=w$ in (2.18), as in the proof of Proposition 2.5, we obtain

$k\|\nabla w\|_{L^{2}}^{2}+\frac{3}{4} \int_{\Omega} b(u+\vartheta) S(u)^{2} w^{2} \leq\|\vartheta\|_{L^{\infty}}\|\nabla S(u)\|_{L^{2}}\|\nabla w\|_{L^{2}}+\left\|S(u)^{3}\right\|_{L^{2}}\|w\|_{L^{2}}\|\vartheta\|_{L^{\infty}}$

and again by Proposition 2.4

$k\|\nabla w\|_{L^{2}}^{2}+\frac{3}{4} m^{2 / 3}\|w\|_{L^{2}\left(\Omega^{d_{0}}\right)}^{2} \leq\|\vartheta\|_{L^{\infty}}\|\nabla S(u)\|_{L^{2}}\|\nabla w\|_{L^{2}}+\|\vartheta\|_{L^{\infty}}\left\|S(u)^{3}\right\|_{L^{2}}\|w\|_{L^{2}}$.

By (2.5) and the Sobolev inequality, eventually

$$
\|w\|_{H^{1}(\Omega)}^{2} \leq C\|S(u)\|_{H^{1}(\Omega)}\|w\|_{H^{1}(\Omega)}\|\vartheta\|_{L^{\infty}}
$$

hence $\|S(u+\vartheta)-S(u)\|_{H^{1}(\Omega)}=O\left(\|\vartheta\|_{L^{\infty}(\Omega)}\right)$.

Take now (2.18) and subtract (2.16). Define $r=S(u+\vartheta)-S(u)-S_{*}$ : it holds that

$$
\begin{aligned}
\int_{\Omega} a(u) \nabla r \nabla \varphi+\int_{\Omega} b(u) 3 S(u)^{2} r \varphi= & \int_{\Omega}(a(u+\vartheta)-a(u)) \nabla w \cdot \nabla \varphi \\
& +\int_{\Omega}\left(b(u+\vartheta) q-3 b(u) S(u)^{2}\right) w \varphi \quad \forall \varphi \in H^{1}(\Omega) .
\end{aligned}
$$

The second integral in the latter sum can be split as follows:

$$
\int_{\Omega}\left(b(u+\vartheta) q-3 b(u) S(u)^{2}\right) w \varphi=\int_{\Omega}(b(u+\vartheta)-b(u)) q w \varphi+\int_{\Omega}\left(q-3 S(u)^{2}\right) b(u) w \varphi,
$$

and in particular $q-3 S(u)^{2}=S(u+\vartheta)^{2}+S(u+\vartheta) S(u)-2 S(u)^{2}=h w$, where $h=S(u+$ $\vartheta)+2 S(u) \in H^{1}(\Omega)$. Hence, chosen $\varphi=r$ and exploiting again Proposition 2.4, the Poincaré inequality in Lemma 2.1 and the Hölder inequality:

$$
\begin{aligned}
\frac{1}{C}\|r\|_{H^{1}}^{2} \leq & k\|\nabla r\|_{L^{2}}^{2}+m^{2 / 3}\|r\|_{L^{2}\left(\Omega^{d_{0}}\right)} \leq(1-k)\|\vartheta\|_{L^{\infty}}\|\nabla w\|_{L^{2}}\|\nabla r\|_{L^{2}} \\
& +\|\vartheta\|_{L^{\infty}}\|q\|_{L^{4}}\|w\|_{L^{2}}\|r\|_{L^{4}}+\|h\|_{L^{4}}\|w\|_{L^{4}}^{2}\|r\|_{L^{4}} \\
& \leq\left((1-k)\|\vartheta\|_{L^{\infty}}\|w\|_{H^{1}}+\|q\|_{H^{1}}\|\vartheta\|_{L^{\infty}}\|w\|_{H^{1}}+\|h\|_{H^{1}}\|w\|_{H^{1}}^{2}\right)\|r\|_{H^{1}}
\end{aligned}
$$


It follows eventually that $\|r\|_{H^{1}(\Omega)} \leq C\|\vartheta\|_{L^{\infty}}^{2}=o\left(\|\vartheta\|_{L^{\infty}}\right)$, which guarantees that $S_{*}=$ $S^{\prime}(u)[\vartheta]$.

The last step is to provide an expression of the Fréchet derivative of $J_{\varepsilon}$. Exploiting the fact that $S$ is differentiable, we can compute the expression of $J_{\varepsilon}^{\prime}(u)$ through the chain rule:

$$
J_{\varepsilon}^{\prime}(u)[\vartheta]=\int_{\partial \Omega}\left(S(u)-y_{0}\right) S^{\prime}(u)[\vartheta]+\alpha \int_{\Omega}\left(2 \varepsilon \nabla u \nabla \vartheta+\frac{1}{\varepsilon}(1-2 u) \vartheta\right) .
$$

Finally, thanks to the expression of the adjoint problem,

$$
\begin{aligned}
\int_{\partial \Omega}\left(S(u)-y_{0}\right) S^{\prime}(u)[\vartheta] & =\int_{\partial \Omega}\left(S(u)-y_{0}\right) S_{*}=\int_{\Omega} a(u) \nabla p \cdot \nabla S_{*}+\int_{\Omega} 3 S(u)^{2} p S_{*}= \\
\left(\text { by definition of } S_{*}\right) & =\int_{\Omega}(1-k) \vartheta \nabla S(u) \cdot \nabla p+\int_{\Omega} \vartheta S(u)^{3} p,
\end{aligned}
$$

and hence:

$$
J_{\varepsilon}^{\prime}(u)[\vartheta]=\int_{\Omega}(1-k) \vartheta \nabla S(u) \cdot \nabla p+\int_{\Omega} \vartheta S(u)^{3} p+\alpha \int_{\Omega}\left(2 \varepsilon \nabla u \cdot \nabla \vartheta+\frac{1}{\varepsilon}(1-2 u) \vartheta\right) .
$$

Finally, it is a standard argument that, being $J_{\varepsilon}$ a continuous and Frechét differentiable functional on a convex subset $\mathcal{K}$ of the Banach space $H^{1}(\Omega)$, the optimality conditions for the optimization problem (2.12) are expressed by the variational inequality (2.13).

3. Discretization and reconstruction algorithm For a fixed $\varepsilon>0$, we now introduce a Finite Element formulation of problem (2.12) in order to define a numerical reconstruction algorithm and compute an approximated solution of the inverse problem.

In what follows, we consider $\Omega$ to be polygonal, in order to avoid a discretization error involving the geometry of the domain. Let $\mathcal{T}_{h}$ be a shape regular triangulation of $\Omega$ and define $V_{h} \subset H^{1}(\Omega)$ :

$$
V_{h}=\left\{v_{h} \in C(\bar{\Omega}),\left.v_{h}\right|_{K} \in \mathbb{P}_{1}(K) \forall K \in \mathcal{T}_{h}\right\} ; \quad \mathcal{K}_{h}=V_{h} \cap \mathcal{K} .
$$

For every fixed $h>0$, we define the solution map $S_{h}: \mathcal{K} \rightarrow V_{h}$, where $S_{h}(u)$ solves

$$
\int_{\Omega} a(u) \nabla S_{h}(u) \nabla v_{h}+\int_{\Omega} b(u) S_{h}(u)^{3} v_{h}=\int_{\Omega} f v_{h} \quad \forall v_{h} \in V_{h} .
$$

3.1. Convergence analysis as $h \rightarrow 0$ The present section is devoted to the numerical analysis of the discretized problem: the convergence of the approximated solution of the direct problem is studied, taking into account the difficulties implied by the nonlinear term. Moreover, the existence and convergence of minimizers of the discrete cost functional is analysed. The following result, which is preliminary for the proof of the convergence of the approximated solutions to the exact one, can be proved by resorting to the techniques of [18, Theorem 2.1].

LEMma 3.1. Let $f \in L^{2}(\Omega)$ satisfy assumption (2.2); then, for every $u \in \mathcal{K}, S_{h}(u) \rightarrow S(u)$ strongly in $H^{1}(\Omega)$. 
Proof. As in the proof of Proposition 2.1, for a fixed $u \in \mathcal{K}$ we define the operator $T: H^{1}(\Omega) \rightarrow\left(H^{1}(\Omega)\right)^{*}$ such that

$$
\langle T(y), \varphi\rangle=\int_{\Omega} a(u) \nabla y \nabla \varphi+\int_{\Omega} b(u) y^{3} \varphi
$$

then $y_{h}=S_{h}(u)$ and $y=S(u)$ are respectively the solutions of the equations

$$
\left\langle T\left(y_{h}\right), \varphi_{h}\right\rangle=\int_{\Omega} f \varphi_{h} \quad \forall \varphi_{h} \in V_{h} ; \quad\langle T(y), \varphi\rangle=\int_{\Omega} f \varphi \quad \forall \varphi \in H^{1}(\Omega) .
$$

The ellipticity of the operator $T$ follows by Lemma 2.1 and Proposition 2.4, indeed:

$$
\begin{aligned}
\left\langle T\left(y_{h}\right)-T(y), y_{h}-y\right\rangle & =\int_{\Omega} a(u)\left|\nabla\left(y_{h}-y\right)\right|^{2}+\int_{\Omega} b(u)\left(y_{h}-y\right)^{2}\left(y_{h}^{2}+y_{h} y+y^{2}\right) \\
& \geq k\left\|\nabla\left(y_{h}-y\right)\right\|_{L^{2}(\Omega)}^{2}+\frac{3}{4} m^{2 / 3}\left\|y y_{h}-y\right\|_{L^{2}\left(\Omega^{\left.d_{0}\right)}\right.}^{2} \geq C\left\|y_{h}-y\right\|_{H^{1}(\Omega)}^{2},
\end{aligned}
$$

where $C=C\left(k, m, \Omega, d_{0}\right)$ is independent of $h$. Consider now an arbitrary $w_{h} \in V_{h}$ and exploit the orthogonality $\left\langle T\left(y_{h}\right)-T(y), \varphi_{h}\right\rangle=0 \forall \varphi_{h} \in V_{h}$, which follows by (3.1).

$$
\begin{aligned}
C\left\|y_{h}-y\right\|_{H^{1}}^{2} & \leq\left\langle T\left(y_{h}\right)-T(y), y_{h}-y\right\rangle=\left\langle T\left(y_{h}\right)-T(y), w_{h}-y\right\rangle \\
& \leq K\left\|w_{h}-y\right\|_{H^{1}}\left\|y_{h}-y\right\|_{H^{1}},
\end{aligned}
$$

where $K$ is the Lipschitz constant of $T$ (see Proposition 2.1). We point out that, in view of Proposition 2.2, the constant $K$ does not depend on $u$ nor on $h$, but only on $\|f\|_{L^{2}(\Omega)}, \Omega, d_{0}, k$. Hence:

$$
\left\|y_{h}-y\right\|_{H^{1}} \leq \frac{K}{C}\left\|w_{h}-y\right\|_{H^{1}}
$$

and since the latter inequality holds for each $w_{h} \in H^{1}(\Omega)$, it holds:

$$
\left\|y_{h}-y\right\|_{H^{1}(\Omega)} \leq \frac{K}{C} \inf _{w_{h} \in V_{h}}\left\|w_{h}-y\right\|_{H^{1}(\Omega)},
$$

and the thesis follows by the interpolation estimates of $H^{1}(\Omega)$ functions in $V_{h}$.

The convergence of the solution of the discrete direct problem to the continuous one is an immediate consequence of Lemma 3.1 and of the continuity of the map $S_{h}$ in the space $V_{h}$, which can be assessed analogously to the proof of Proposition 2.5.

Proposition 3.1. Let $\left\{h_{k}\right\},\left\{u_{k}\right\}$ be two sequences such that $h_{k} \rightarrow 0, u_{k} \in \mathcal{K}_{h_{k}}$ and $u_{k} \stackrel{L^{1}}{\rightarrow} u \in \mathcal{K}$. Then $S_{h_{k}}\left(u_{k}\right) \stackrel{H^{1}}{\longrightarrow} S(u)$.

Define the discrete cost functional, $J_{\varepsilon, h}: \mathcal{K}_{h} \rightarrow \mathbb{R}$

$$
J_{\varepsilon, h}\left(u_{h}\right)=\frac{1}{2}\left\|S_{h}\left(u_{h}\right)-y_{\text {meas }, h}\right\|_{L^{2}(\partial \Omega)}^{2}+\alpha \int_{\Omega}\left(\varepsilon\left|\nabla u_{h}\right|^{2}+\frac{1}{\varepsilon} u_{h}\left(1-u_{h}\right)\right),
$$

being $y_{\text {meas }, h}$ the $L^{2}(\Omega)$-projection of the boundary datum $y_{\text {meas }}$ in the space of the traces of $V_{h}$ functions. The existence of minimizers of the discrete functionals $J_{\varepsilon, h}$ is stated in the following proposition, together with an asymptotic analysis as $h \rightarrow 0$. 
Taking advantage of Proposition 3.1, the proof is analogous to the one of [20, Theorem $3.2]$.

Proposition 3.2. For each $h>0$, there exists $u_{h} \in \mathcal{K}_{h}$ such that $J_{\varepsilon, h}\left(u_{h}\right)=$ $\min _{v_{h} \in \mathcal{K}_{h}} J_{\varepsilon, h}\left(v_{h}\right)$. Every sequence $\left\{u_{h_{k}}\right\}$ s.t. $\lim _{k \rightarrow \infty} h_{k}=0$ admits a subsequence that converges in $H^{1}(\Omega)$ to a minimum of the cost functional $J_{\varepsilon}$.

The strategy we adopt in order to minimize the discrete cost functional $J_{\varepsilon, h}$ is to search for a function $u_{h}$ satisfying discrete optimality conditions, which can be obtained as in section 2.1:

$$
J_{\varepsilon, h}^{\prime}\left(u_{h}\right)\left[v_{h}-u_{h}\right] \geq 0 \quad \forall v_{h} \in \mathcal{K}_{h}
$$

where for each $\theta_{h} \in \mathcal{K}_{h}-u_{h}:=\left\{\theta_{h}=v_{h}-u_{h} ; v_{h} \in \mathcal{K}_{h}\right\}$ it holds

$$
\begin{aligned}
J_{\varepsilon, h}^{\prime}\left(u_{h}\right)\left[\vartheta_{h}\right]= & \int_{\Omega}(1-k) \vartheta_{h} \nabla S_{h}\left(u_{h}\right) \cdot \nabla p_{h}+\int_{\Omega} \vartheta_{h} S_{h}\left(u_{h}\right)^{3} p_{h}+2 \alpha \varepsilon \int_{\Omega} \nabla u_{h} \cdot \nabla \vartheta_{h} \\
& +\frac{\alpha}{\varepsilon} \int_{\Omega}\left(1-2 u_{h}\right) \vartheta_{h},
\end{aligned}
$$

where $p_{h}$ is the solution in $V_{h}$ of the adjoint problem (2.15) associated to $u_{h}$.

It is finally possible to demonstrate the convergence of critical points of the discrete functionals $J_{\varepsilon, h}$ (i.e., functions in $\mathcal{K}_{h}$ satisfying (3.3)) to a critical point of the continuous one, $J_{\varepsilon}$. The proof can be adapted from the one of [20, Theorem 3.2].

Proposition 3.3. Consider a sequence $\left\{h_{k}\right\}$ s.t. $h_{k} \rightarrow 0$ and for every $k$ denote as $u_{k}$ a solution of the discrete variational inequality (3.3). Then there exists a subsequence of $\left\{u_{k}\right\}$ that converges a.e and in $H^{1}(\Omega)$ to a solution $u$ of the continuous variational inequality (2.14)

\subsection{Reconstruction algorithm: a Parabolic Obstacle Problem approach}

The necessary optimality conditions that have been stated in Proposition 2.10, together with the expression of the Fréchet derivative of the cost functional reported in (2.14) allow to define a Parabolic Obstacle problem, which consists in a very common strategy in order to search for a solution of optimization problems in a phase-field approach. In this section we give a continuous formulation of the problem, and provide a formal proof of its desired properties. We then introduce a numerical discretization of the problem and rigorously prove the main convergence results.

The core of the proposed approach is to rely on a parabolic problem whose solution $u(\cdot, t)$ converges, as the fictitious time variable tends to $+\infty$, to an asymptotic state $u_{\infty}$ satisfying the continuous optimality conidtions (2.14). The problem can be formulated as follows, for a fixed $\varepsilon>0$ : let $u$ be the solution of

$$
\left\{\begin{array}{c}
\int_{\Omega} \partial_{t} u(v-u)+J_{\varepsilon}^{\prime}(u)[v-u] \geq 0 \quad \forall v \in \mathcal{K}, \quad t \in(0,+\infty) \\
u(\cdot, 0)=u_{0} \in \mathcal{K} .
\end{array}\right.
$$

The theoretical analysis of the latter problem is beyond the purposes of this work, and would require to deal with the severe nonlinearity of the expression of $J_{\varepsilon}^{\prime}(u)$; consequently, we provide a complete discretization of the Parabolic Obstacle Problem and 
assess its convergence properties. This is performed by setting (3.5) in the discrete spaces $\mathcal{K}_{h}$ and $V_{h}$, and by considering a semi-implicit one-step scheme for the time updating, as in [20]: i.e., by treating explicitly the nonlinear terms and implicitly the linear ones. We obtain that the approximate solution $\left\{u_{h}^{n}\right\}_{n \in \mathbb{N}} \subset V_{h}, u_{h}^{n} \approx u\left(\cdot, t^{n}\right)$ is computed as:

$$
\left\{\begin{array}{r}
u_{h}^{0}=u_{0} \in \mathcal{K}_{h} \quad \text { (a prescribed initial datum) } \\
u_{h}^{n+1} \in \mathcal{K}_{h}: \int_{\Omega}\left(u_{h}^{n+1}-u_{h}^{n}\right)\left(v_{h}-u_{h}^{n+1}\right)+\tau_{n} \int_{\Omega}(1-k) \nabla S_{h}\left(u_{h}^{n}\right) \cdot \nabla p_{h}^{n}\left(v_{h}-u_{h}^{n+1}\right) \\
\quad+\tau_{n} \int_{\Omega} S_{h}\left(u_{h}^{n}\right)^{3} p_{h}^{n}\left(v_{h}-u_{h}^{n+1}\right)+2 \tau_{n} \alpha \varepsilon \int_{\Omega} \nabla u_{h}^{n+1} \cdot \nabla\left(v_{h}-u_{h}^{n+1}\right) \\
\quad+\tau_{n} \alpha \frac{1}{\varepsilon} \int_{\Omega}\left(1-2 u_{h}^{n}\right)\left(v_{h}-u_{h}^{n+1}\right) \geq 0 \quad \forall v_{h} \in \mathcal{K}_{h}, n=0,1, \ldots
\end{array}\right.
$$

The following preliminary result is necessary for the proof of the convergence of the algorithm:

LEMMA 3.2. For each $n>0$, there exists a positive constant $\mathcal{B}_{n}=$ $\mathcal{B}_{n}\left(\Omega, h, k,\left\|p_{h}^{n}\right\|_{H^{1}},\left\|y_{h}^{n}\right\|_{H^{1}},\left\|y_{h}^{n+1}\right\|_{H^{1}}\right)$ such that, provided that $\tau_{n} \leq \mathcal{B}_{n}$ it holds that:

$$
\left\|u_{h}^{n+1}-u_{h}^{n}\right\|_{L^{2}}^{2}+J_{\varepsilon, h}\left(u_{h}^{n+1}\right) \leq J_{\varepsilon, h}\left(u_{h}^{n}\right) \quad n>0 .
$$

Proof. In the expression of the discrete parabolic obstacle problem (3.6), consider $v_{h}=u_{h}^{n}$ : via simple computation, we can point out that

$$
\begin{aligned}
& \frac{1}{\tau_{n}}\left\|u_{h}^{n+1}-u_{h}^{n}\right\|_{L^{2}}^{2}+J\left(u_{h}^{n+1}\right)-J\left(u_{h}^{n}\right)+\alpha \varepsilon\left\|\nabla\left(u_{h}^{n+1}-u_{h}^{n}\right)\right\|_{L^{2}}^{2}+\frac{\alpha}{\varepsilon}\left\|u_{h}^{n+1}-u_{h}^{n}\right\|_{L^{2}}^{2} \\
& \quad \leq \int_{\Omega}\left(a\left(u_{h}^{n+1}\right)-a\left(u_{h}^{n}\right)\right) \nabla y_{h}^{n} \nabla p_{h}^{n}+\int_{\Omega}\left(b\left(u_{h}^{n+1}\right)-b\left(u_{h}^{n}\right)\right)\left(y_{h}^{n}\right)^{3} p_{h}^{n} \\
& \quad+\frac{1}{2}\left\|y_{h}^{n+1}-y_{h}^{n}\right\|_{L^{2}(\partial \Omega)}^{2}+\int_{\partial \Omega}\left(y_{h}^{n+1}-y_{h}^{n}\right)\left(y_{h}^{n+1}-y_{\text {meas }, h}\right),
\end{aligned}
$$

where $y_{h}^{n}=S_{h}\left(u_{h}^{n}\right)$ and $y_{h}^{n+1}=S_{h}\left(u_{h}^{n+1}\right)$. Moreover, by the expression of the adjoint problem,

$$
R H S=\frac{1}{2}\left\|y_{h}^{n+1}-y_{h}^{n}\right\|_{L^{2}(\partial \Omega)}^{2}+\text { I }+ \text { II }
$$

where

$$
\begin{aligned}
\mathrm{I}= & \int_{\Omega}\left(a\left(u_{h}^{n+1}\right)-a\left(u_{h}^{n}\right)\right) \nabla y_{h}^{n} \cdot \nabla p_{h}^{n}+\int_{\Omega} a\left(u_{h}^{n}\right) \nabla p_{h}^{n} \cdot \nabla\left(y_{h}^{n+1}-y_{h}^{n}\right) \\
= & \int_{\Omega}\left(a\left(u_{h}^{n}\right)-a\left(u_{h}^{n+1}\right)\right) \nabla\left(y_{h}^{n+1}-y_{h}^{n}\right) \cdot \nabla p_{h}^{n}+\int_{\Omega} a\left(u_{h}^{n+1}\right) \nabla y_{h}^{n+1} \cdot \nabla p_{h}^{n} \\
& -\int_{\Omega} a\left(u_{h}^{n}\right) \nabla y_{h}^{n} \cdot \nabla p_{h}^{n} ;
\end{aligned}
$$




$$
\begin{aligned}
\text { II }= & \int_{\Omega}\left(b\left(u_{h}^{n+1}\right)-b\left(u_{h}^{n}\right)\right)\left(y_{h}^{n}\right)^{3} p_{h}^{n}+3 \int_{\Omega} b\left(u_{h}^{n}\right)\left(y_{h}^{n}\right)^{2} p_{h}^{n}\left(y_{h}^{n+1}-y_{h}^{n}\right)= \\
= & \int_{\Omega} b\left(u_{h}^{n+1}\right)\left(\left(y_{h}^{n}\right)^{3}-\left(y_{h}^{n+1}\right)^{3}\right) p_{h}^{n}+3 \int_{\Omega} b\left(u_{h}^{n}\right)\left(y_{h}^{n}\right)^{2} p_{h}^{n}\left(y_{h}^{n+1}-y_{h}^{n}\right) \\
& +\int_{\Omega} b\left(u_{h}^{n+1}\right)\left(y_{h}^{n+1}\right)^{3} p_{h}^{n}-\int_{\Omega} b\left(u_{h}^{n}\right)\left(y_{h}^{n}\right)^{3} p_{h}^{n}= \\
& \left(\text { by the expansion }\left(y_{h}^{n+1}\right)^{3}=\left(y_{h}^{n}+\left(y_{h}^{n+1}-y_{h}^{n}\right)\right)^{3}\right) \\
= & 3 \int_{\Omega}\left(b\left(u_{h}^{n}\right)-b\left(u_{h}^{n+1}\right)\right)\left(y_{h}^{n}\right)^{2} p_{h}^{n}\left(y_{h}^{n+1}-y_{h}^{n}\right)-3 \int_{\Omega} b\left(u_{h}^{n+1}\right)\left(y_{h}^{n}\right) p_{h}^{n}\left(y_{h}^{n+1}-y_{h}^{n}\right)^{2} \\
& -\int_{\Omega} b\left(u_{h}^{n+1}\right) p_{h}^{n}\left(y_{h}^{n+1}-y_{h}^{n}\right)^{3}+\int_{\Omega} b\left(u_{h}^{n+1}\right)\left(y_{h}^{n+1}\right)^{3} p_{h}^{n}-\int_{\Omega} b\left(u_{h}^{n}\right)\left(y_{h}^{n}\right)^{3} p_{h}^{n} .
\end{aligned}
$$

Collecting the terms and taking advantage of the expression of the direct problem, we conclude that

$$
\begin{aligned}
R H S=\frac{1}{2}\left\|y_{h}^{n+1}-y_{h}^{n}\right\|_{L^{2}(\partial \Omega)}^{2}+\int_{\Omega}\left(a\left(u_{h}^{n}\right)-a\left(u_{h}^{n+1}\right)\right) \nabla\left(y_{h}^{n+1}-y_{h}^{n}\right) \cdot \nabla p_{h}^{n} \\
+3 \int_{\Omega}\left(b\left(u_{h}^{n}\right)-b\left(u_{h}^{n+1}\right)\right)\left(y_{h}^{n}\right)^{2} p_{h}^{n}\left(y_{h}^{n+1}-y_{h}^{n}\right) \\
\quad-3 \int_{\Omega} b\left(u_{h}^{n+1}\right)\left(y_{h}^{n}\right) p_{h}^{n}\left(y_{h}^{n+1}-y_{h}^{n}\right)^{2}-\int_{\Omega} b\left(u_{h}^{n+1}\right) p_{h}^{n}\left(y_{h}^{n+1}-y_{h}^{n}\right)^{3} .
\end{aligned}
$$

We now employ the Cauchy-Schwarz inequality and the regularity of the solutions of the discrete direct and adjoint problems (in particular the equivalence of the $W^{1, \infty}$ and $H^{1}$ norm in $\left.V_{h}:\left\|u_{h}\right\|_{W^{1, \infty}} \leq C_{1}\left\|u_{h}\right\|_{H^{1}}, C_{1}=C_{1}(\Omega, h)\right)$ :

$$
R H S \leq C_{2}\left\|u_{h}^{n+1}-u_{h}^{n}\right\|_{L^{2}}\left\|y_{h}^{n+1}-y_{h}^{n}\right\|_{H^{1}}+C_{3}\left\|y_{h}^{n+1}-y_{h}^{n}\right\|_{H^{1}}^{2}
$$

with $\quad C_{2}=(1-k) C_{1}\left\|p_{h}^{n}\right\|_{H^{1}}+C_{1}\left\|y_{h}^{n}\right\|_{H^{1}}\left\|p_{h}^{n}\right\|_{H^{1}} \quad$ and $\quad C_{3}=3 C_{1}^{2}\left\|y_{h}^{n}\right\|_{H_{1}}\left\|p_{h}^{n}\right\|_{H_{1}}+$ $C_{1}^{3}\left\|p_{h}^{n}\right\|_{H_{1}}\left(\left\|y_{h}^{n}\right\|_{H_{1}}+\left\|y_{h}^{n+1}\right\|_{H_{1}}\right)+\frac{1}{2} C_{t r}^{2}$, being $C_{t r}$ the constant of the trace inequality in $H^{1}(\Omega)$. Eventually, similarly to the computation included in the proof of Proposition 2.10, one can assess that

$$
\left\|y_{h}^{n+1}-y_{h}^{n}\right\|_{H^{1}} \leq C_{4}\left\|u_{h}^{n+1}-u_{h}^{n}\right\|_{L^{2}},
$$

with $C_{4}=C_{4}\left(k, C_{1},\left\|y_{h}^{n}\right\|_{H^{1}}, \Omega\right)$. Hence, we can conclude that there exists a positive constant $\mathcal{C}_{n}=C_{2} C_{4}+C_{3} C_{4}^{2}$ such that

$$
\frac{1}{\tau_{n}}\left\|u_{h}^{n+1}-u_{h}^{n}\right\|_{L^{2}}^{2}+J\left(u_{h}^{n+1}\right)-J\left(u_{h}^{n}\right) \leq \mathcal{C}_{n}\left\|u_{h}^{n+1}-u_{h}^{n}\right\|_{L^{2}}^{2},
$$

and choosing $\tau_{n}\left\langle\mathcal{B}_{n}:=\frac{1}{1+\mathcal{C}_{n}}\right.$ we can conclude the thesis.

We are finally able to prove the following convergence result for the fully discretized Parabolic Obstacle Problem:

Proposition 3.4. Consider a starting point $u_{h}^{0} \in \mathcal{K}_{h}$. Then, there exists a collection of timesteps $\left\{\tau_{n}\right\}$ s.t. $0<\gamma \leq \tau_{n} \leq \mathcal{B}_{n} \forall n>0$. Corresponding to $\left\{\tau_{n}\right\}$, the sequence $\left\{u_{h}^{n}\right\}$ generated by (3.6) has a converging subsequence (which we still denote with $u_{h}^{n}$ ) such that $u_{h}^{n} \stackrel{W^{1, \infty}}{\longrightarrow} u_{h} \in V_{h}$, which satisfies the discrete optimality conditions (3.3). 
Proof. Consider a generic collection of timesteps $\tilde{\tau}_{n}$ satisfying $\tilde{\tau}_{n} \leq \mathcal{B}_{n} \forall n>0$. Hence, by Lemma 3.2,

$$
\sum_{n=0}^{\infty}\left\|u_{h}^{n+1}-u_{h}^{n}\right\|_{L^{2}}^{2} \leq J_{\varepsilon, h}\left(u_{h}^{0}\right) \quad \text { and } \quad \sup _{n} J_{\varepsilon, h}\left(u_{h}^{n}\right) \leq J_{\varepsilon, h}\left(u_{h}^{0}\right)
$$

which implies that $\left\|u_{h}^{n+1}-u_{h}^{n}\right\|_{L^{2}} \rightarrow 0$ and hence $u_{h}^{n}$ is bounded in $H^{1}(\Omega)$, and this implies that also $\left\{y_{h}^{n}\right\}$ and $\left\{p_{h}^{n}\right\}$ are bounded in $H^{1}(\Omega)$. According to the definition of the constants $\mathcal{C}_{n}$ and $\mathcal{B}_{n}$ reported in the proof of Lemma 3.2, this entails that there exists a constant $M>0$ such that $\mathcal{C}_{n} \leq M \forall n>0$, and equivalently there exists a positive constant $\gamma$ s.t. $\gamma \leq \mathcal{B}_{n}$. Hence, it is possible to choose, for each $n>0, \gamma \leq \tau_{n} \leq \mathcal{B}_{n}$.

Eventually, we conclude that there exists $u_{h} \in \mathcal{K}_{h}$ such that, up to a subsequence, $u_{h}^{n} \rightarrow$ $u_{h}$ a.e. and in $W^{1, \infty}(\Omega)$ (and $y_{h}^{n} \rightarrow y_{h}:=S_{h}\left(u_{h}\right), p_{h}^{n} \rightarrow p_{h}$ in $H^{1}$ and in $W^{1, \infty}$ as well, as in the discrete space $V_{h}$ the $L^{\infty}$ norm is equivalent to the $L^{2}(\Omega)$ ). We exploit the expression of the discrete Parabolic Obstacle Problem (3.6) to show that

$$
\begin{array}{r}
\int_{\Omega}(1-k) \nabla y_{h}^{n} \cdot \nabla p_{h}^{n}\left(v_{h}-u_{h}^{n+1}\right)+\int_{\Omega}\left(y_{h}^{n}\right)^{3} p_{h}^{n}\left(v_{h}-u_{h}^{n+1}\right)+2 \alpha \varepsilon \int_{\Omega} \nabla u_{h}^{n+1} \cdot \nabla\left(v_{h}-u_{h}^{n+1}\right) \\
+\alpha \frac{1}{\varepsilon} \int_{\Omega}\left(1-2 u_{h}^{n}\right)\left(v_{h}-u_{h}^{n+1}\right) \geq-\frac{1}{\tau_{n}} \int_{\Omega}\left(u_{h}^{n+1}-u_{h}^{n}\right)\left(v_{h}-u_{h}^{n+1}\right) \quad \forall v_{h} \in \mathcal{K}_{h},
\end{array}
$$

and since $-\frac{1}{\tau_{n}}>-\frac{1}{\gamma} \forall n$, when taking the limit as $n \rightarrow \infty$, the right-hand side converges to 0 , which entails that $u_{h}$ satisfies the discrete optimality conditions (3.3).

In order to solve (3.6) we resort the Primal-Dual Active Set method, introduced in [11]. Thus, the final formulation of the reconstruction algorithm is the following:

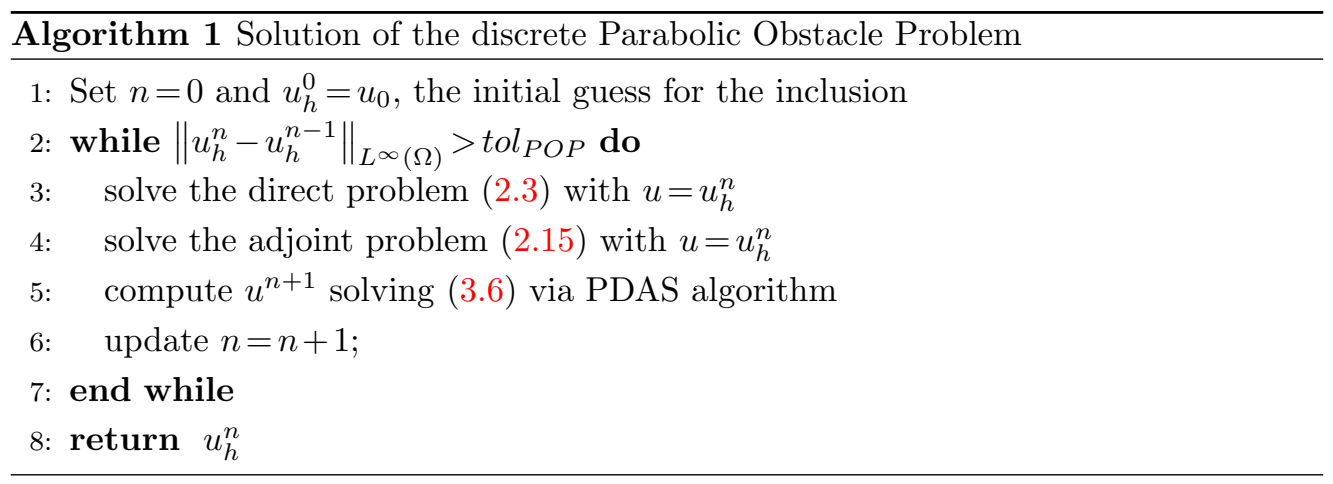

REMARK 3.1. It is a common practice to increase the performance of a reconstruction algorithm taking advantage of multiple measurements. In this context, it is possible to suppose the knowledge of $N_{f}$ different measurements of the electric potential on the boundary, $y_{\text {meas }, j} j=1, \cdots, N_{f}$, associated to different source terms $f_{j}$. Therefore, instead of tackling the optimization of the mismatch functional $J$ as in (2.10), it is possible to introduce the averaged cost functional $J^{T O T}(u)=\frac{1}{N_{f}} \sum_{j=1}^{N_{f}} J^{j}(u)$, where $J^{j}(u)=\frac{1}{2}\left\|S_{j}(u)-y_{\text {meas }, j}\right\|_{L^{2}(\partial \Omega)}^{2}$, being $S_{j}(u)$ the solution of the direct problem $(2.3)$ 
with source term $f=f_{j}$. The process of regularization, relaxation and computation of the optimality conditions is exactly the same as for $J$, and yields the same reconstruction algorithm as in Algorithm 1, where at each timestep the solution of $N^{f}$ direct and adjoint problem must be computed.

4. Numerical results In this section we report various results obtained applying Algorithm 1. In all the numerical experiments, we consider $\Omega=(-1,1)^{2}$ and we introduce an uniform and shape regular tessellation $\mathcal{T}_{h}$ of triangles. Due to the lack of experimental measures of the boundary datum $y_{\text {meas }}$, we make use of synthetic data, i.e., we simulate the direct problem via the Finite Element method, considering the presence of an ischemic region of prescribed geometry, and extract the value on the boundary of the domain. In order to avoid to incurr an inverse crime (i.e. the performance of the reconstruction algorithm are improved by the fact that the exact data are synthetically generated with the same numerical scheme adopted in the algorithm) we introduce a more refined mesh $\mathcal{T}_{h}^{e x}$ on which the exact problem is solved, and interpolate the resulting datum $y_{\text {meas }}$ on the mesh $\mathcal{T}_{h}$.

In the following test cases, we apply Algorithm 1 for the reconstruct inclusions of different geometries, in order to investigate the effectiveness of the introduced strategy. We use the same computational mesh $\mathcal{T}_{h}$ (mesh size $h=0.04$, nearly 6000 elements) for the numerical solution of the boundary value problems involved in the procedure, except for the generation of each synthetic data which is performed on different finer meshes $\mathcal{T}_{h}^{e x}$. According to Remark 3.1, we make use of $N_{f}=2$ different measurements, associated to the source terms $f_{1}(x, y)=x$ and $f_{2}(x, y)=y$. The main parameters for all the simulations lie in the ranges reported in Table 4.1. We make use of the same relationship between $\varepsilon$ and $\tau$ as in [20]. The initial guess for each simulation is $u_{0} \equiv 0$.

\begin{tabular}{c|c|c|c}
$\alpha$ & $\varepsilon$ & $\tau$ & tol $_{P O P}$ \\
\hline $10^{-4} \div 10^{-3}$ & $1 /(8 \pi)$ & $(0.01 \div 0.1) / \varepsilon$ & $10^{-4}$
\end{tabular}

Table 4.1: Range of the main parameters

In Figure 4.1 we report some of the iterations of Algorithm 1 for the reconstruction of a circular inclusion $(\alpha=0.0001, \tau=0.01 / \varepsilon)$. The boundary $\partial \omega$ is marked with a black line, which is superimposed to the contour plot of the approximation of the indicator function $u_{h}^{n}$ at different timesteps $n$. The algorithm converged after $N_{\text {tot }}=568$ iterations, corresponding to a final (fictitious) time $T_{t o t}=1427.54$. In Figure 4.2 we investigate the effectiveness of the algorithm to reconstruct inclusions of rather complicated geometry. For each test case, we show the contour plot of the final iteration of the reconstruction (the total number of iterations $N$ and the final time $T$ are reported in the caption), and the boundary of the exact inclusion is overlaid in black line. Moreover, each result is equipped with the graphic (in semilogarithmic scale) of the evolution of the cost functional $J_{\varepsilon}$, split into the components $J_{P D E}(u)=\frac{1}{2}\left\|S(u)-y_{\text {meas }}\right\|_{L^{2}(\partial \Omega)}^{2}$ and 


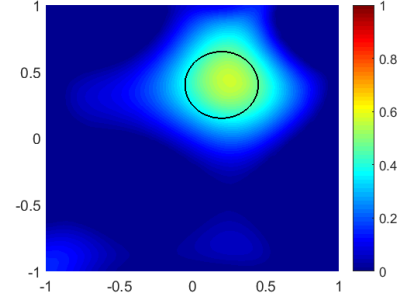

(a) $n=30$

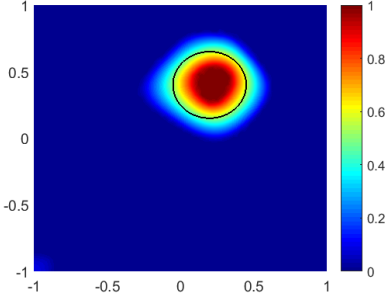

(b) $n=90$

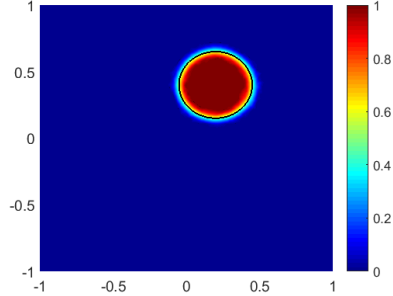

(c) $n=568$

Figure 4.1: Reconstruction of a circular inclusion: successive iterations

$J_{\text {regularization }}(u)=\alpha \varepsilon\|\nabla u\|_{L^{2}(\Omega)}^{2}+\frac{\alpha}{\varepsilon} \int_{\Omega} u(1-u)$. The reported results consist in approx-

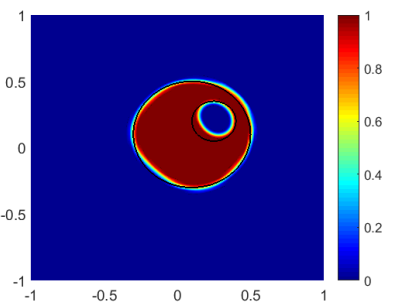

(a) $\quad N_{\text {tot }}=3491, \quad T_{\text {tot }}=3509.54,(\mathrm{~b})$ $\alpha=0.001, \tau=0.02 / \varepsilon$

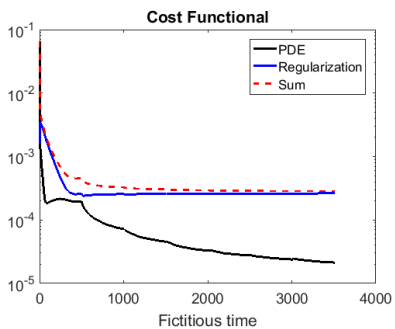

(d) Ellipse: evolution of $J_{\varepsilon}$
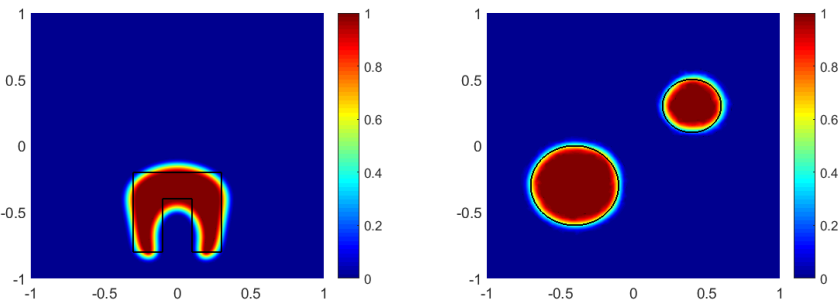

(b) $\quad N_{\text {tot }}=1537, \quad T_{\text {tot }}=772.58,(\mathrm{c}) \quad N_{\text {tot }}=4670, \quad T_{\text {tot }}=2347.40$ $\alpha=0.0001, \tau=0.02 / \varepsilon$ $\alpha=0.0001, \tau=0.02 / \varepsilon$

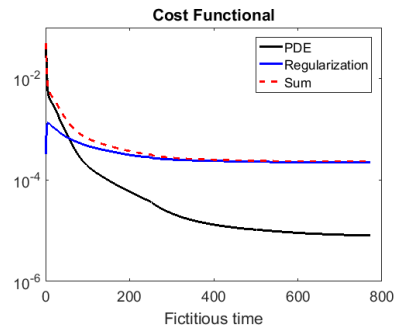

(e) Rectangle: evolution of $J_{\varepsilon}$

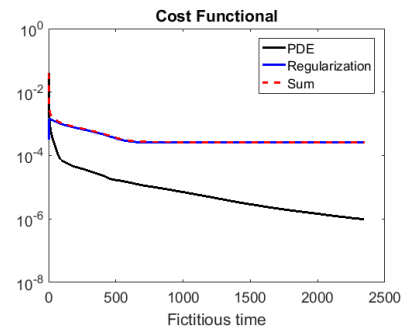

(f) Two circles: evolution of $J_{\varepsilon}$

Figure 4.2: Reconstruction of various inclusions

imations of minimizers of $J_{\varepsilon}$ in $\mathcal{K}$ : they are smooth function and range between 0 and 1. They show large regions in which they attain the limit values 0 and 1 , and a region of diffuse interface between them, whose thickness is about $\varepsilon / 2$. As Figures 4.1 and 4.2 show, the algorithm is able to reconstruct inclusion of rather complicated geometry. The identification of smooth inclusion is performed with higher precision, whereas it seems that the accuracy is low in presence of sharp corners. We point out that we do not need to have any a priori knowledge on the topology of the inclusion $\omega$, i.e., the 
number of connected components is correctly identified.

We now assess that the final result of the reconstruction is independent of the initial guess imposed as a starting point of the Parabolic Obstacle problem. In Figure 4.3 we compare the behaviour of the algorithm applied to the reconstruction of a circular inclusion (the same as in Figure 4.1), where we impose a different initial datum with respect to the constant zero function. In the first experiment, we start from an initial datum which is the indicator function of an arbitrarily chosen region. In the second one, we impose as a starting point the indicator function of a sublevel of the topological gradient of the cost functional $J$. As investigated in [10], the topological gradient is a powerful tool for the detection of small-size inclusions, which yield a small perturbation in the cost functional with respect to the background (unperturbed) case. The position of a small inclusion is easily identified by searching for the point where the topological gradient of $J$ attains its (negative) minimum. As the information given by the topological gradient $G$ has shown to be useful even in the case of large-size inclusions (see, e.g., [7], [14]), we take advantage of it by computing $G$ (see Theorem 3.1 in [10]), setting a threshold $G_{t h r}$ and defining $u_{0}=\chi_{\left\{G \leq G_{t h r}\right\}}$. The results reported in Figure 4.3 show the

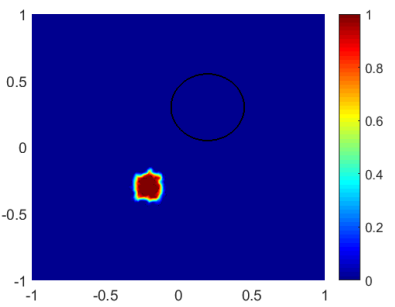

(a) Initial guess: arbitrary

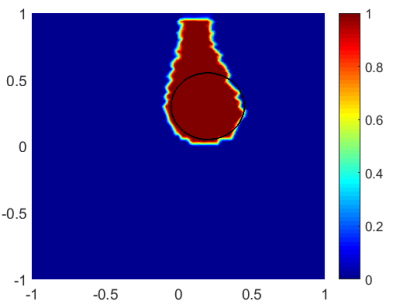

(d) Initial guess: topological

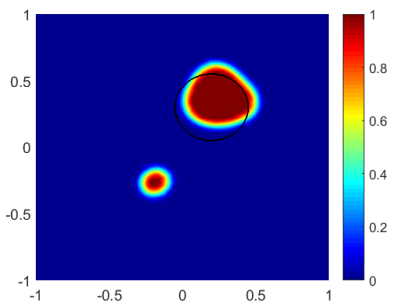

(b) Intermediate: $n=60$

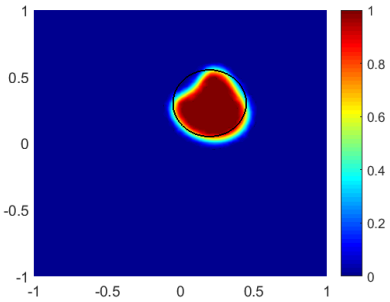

(e) Intermediate: $n=50$

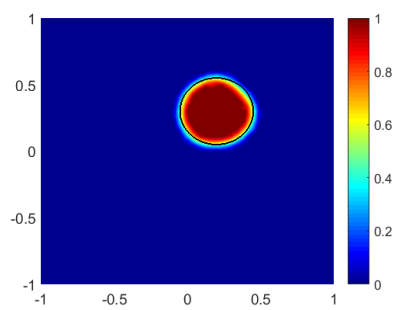

(c) Final: $N_{\text {tot }}=661, T_{\text {tot }}=1661.27$

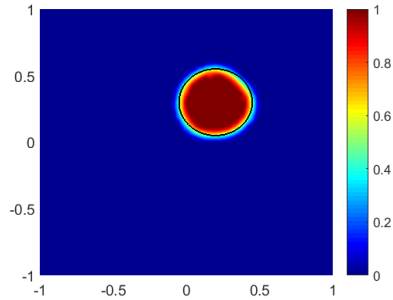

(f) Final: $N_{\text {tot }}=489, T_{\text {tot }}=1228.99$

Figure 4.3: Reconstruction of a circular inclusion with different initial conditions

starting point of the algorithm, an intermediate iteration and the final reconstruction. In both cases we set $\alpha=0.001, \varepsilon=1 /(8 \pi)$ and $\tau=0.1 / \varepsilon$. We underline that the result in each case is similar to the one depicted in Figure 4.1, but through the second strategy it was possible to perform a smaller number of iterations.

We finally verify the stability result obtained in Proposition 2.7, by testing the 
reconstruction algorithm when the measured boundary data are perturbed by a small amount of noise. In particular, we consider $y_{p}=y_{\text {meas }}+p \eta$, being $\eta$ a Gaussian random variable with null mean and standard deviation equal to $\max _{\Omega} y_{\text {meas }}-\min _{\Omega} y_{\text {meas }}$ and $p \in[0,1]$ the noise level. In Figure 4.4 we report the final results of the reconstruction algorithm when applied to the boundary measurements related to an elliptical inclusion perturbed with different noise level. For each simulation, we fix $\alpha=0.001$ and $\varepsilon=\frac{1}{8 \pi}$. In Figure 4.5, instead, we investigate the effect of the regularization parameter $\alpha$ in

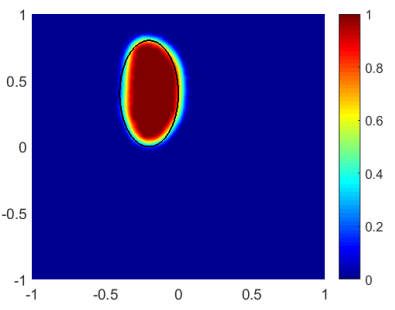

(a) $p=0.01 ; N_{\text {tot }}=430$

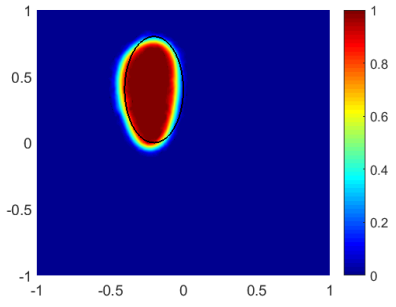

(b) $p=0.05 ; N_{t o t}=503$

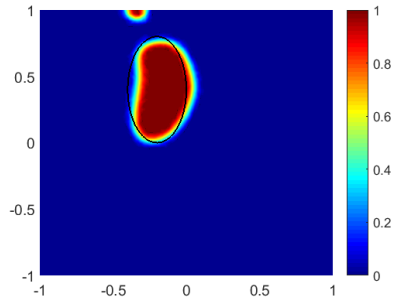

(c) $p=0.1 ; N_{t o t}=1120$

Figure 4.4: Reconstruction of an elliptical inclusion with noisy measurements

the reconstruction from noisy data, fixing $p=0.1$. We observe that a higher value of $\alpha$ may help in filtering the information coming from the noise, avoiding to let it spoil the reconstruction, although it might result in an overall loss of precision.

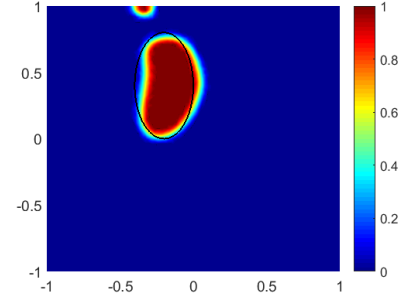

(a) $\alpha=0.001 ; N_{t o t}=1120$

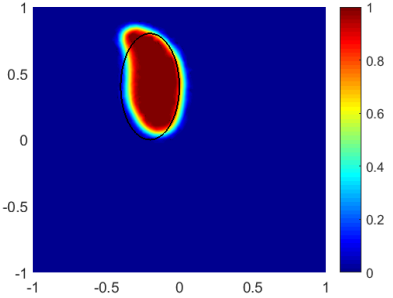

(b) $\alpha=0.003 ; N_{t o t}=751$

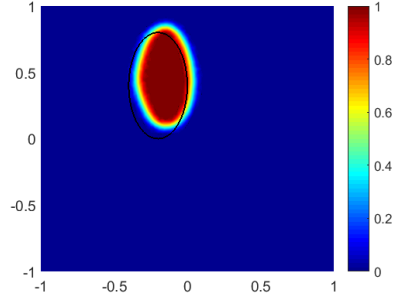

(c) $\alpha=0.01 ; N_{t o t}=462$

Figure 4.5: Reconstruction of an elliptical inclusion with noisy measurements

5. Comparison with the Shape Derivative approach In the previous sections, we have analyzed in detail the phase-field relaxation of the minimization problem expressed in (2.11). We now aim at describing the relationship between this method and a shape derivative based approach, which consists in updating the shape of the inclusion to be reconstructed by perturbing its boundary along the directions of the vector field which causes the greatest descent of the cost functional. Such a direction can be deduced by computing the shape derivative of the functional itself. In this section, we 
first theoretically investigate the relationship between the shape derivative of the cost functional $J_{r e g}$ and the Fréchet derivative of $J_{\varepsilon}$ and then report a comparison between the numerical results of the two algorithms in a set of benchmark cases.

5.1. Sharp interface limit of the Optimality Conditions In order to study the relationship between the optimality conditions in the phase-field approach and the ones derived in the sharp case, we follow an analogous approach as in [12]. First of all, in Proposition 5.1 we introduce the necessary optimality condition for the sharp problem (2.11), taking advantage of the computation of the material derivative of the cost functional. We then define in Proposition 5.3 similar optimality conditions for the relaxed problem (2.12), which are related but not equivalent to the one stated in (2.13)-(2.14) through the Fréchet derivative. In Proposition 5.4 we finally assess the convergence of the phase-field optimality condition to the sharp one when $\varepsilon \rightarrow 0$.

For the sake of simplicity, in this section we will refer to $J_{r e g}$ as $J$. Consider the minimization problem (as in (2.11)):

$$
\underset{u \in X_{0,1}}{\operatorname{argmin}} J(u) ; \quad J(u)=\frac{1}{2}\left\|S(u)-y_{m e a s}\right\|_{L^{2}(\partial \Omega)}^{2}+\alpha T V(u) .
$$

Since $u \in X_{0,1}$ implies that $u=\chi_{\omega}$, being $\omega$ a finite-perimeter subset of $\Omega$, we can perturb $u$ by means of a vector field $\phi_{t}: \Omega \rightarrow \mathbb{R}^{2}, \phi_{t}(x)=x+t V(x)$, being

$$
V \in C^{1}(\Omega) \text { s.t. } V(x)=0 \text { in } \Omega^{d_{0}}=\left\{x \in \Omega \text { s.t. } \operatorname{dist}(x, \partial \Omega) \leq d_{0}\right\} .
$$

Consider the family of functions $\left\{u_{t}\right\}: u_{t}=u \circ \phi_{t}^{-1}$ : we can compute the shape derivative of the functional $J$ in $u$ along the direction $V$ (see [21]) as

$$
D J(u)[V]:=\lim _{t \rightarrow 0} \frac{J\left(u_{t}\right)-J(u)}{t},
$$

where $J\left(u_{t}\right)$ is the cost functional evaluated in the deformed domain $\Omega_{t}=\phi_{t}(\Omega)$ but, according to (5.2), $\Omega_{t}$ and $\Omega$ are the same set, thus we do not adopt a different notation. We prove the following result:

Proposition 5.1. If $u$ is a solution of (5.1) and $f \in L^{2}(\Omega)$ satisfies assumption (2.2), then

$$
D J(u)[V]=0 \quad \text { for all the smooth vector fields } V .
$$

The shape derivative is given by:

$$
D J(u)[V]=\int_{\partial \Omega}\left(S(u)-y_{\text {meas }}\right) \dot{S}(u)[V]+\int_{\Omega}(\operatorname{div} V-D V \nu \cdot \nu) d|D u|,
$$

where $d|D u|=\delta_{\partial \omega} d x, \nu$ is the generalized unit normal vector (see [27]) and $\dot{S}(u)[V]=: \dot{S}$, the material derivative of the solution map, solves

$$
\begin{gathered}
\int_{\Omega} a(u) \nabla \dot{S} \cdot \nabla v+\int_{\Omega} b(u) 3 S(u)^{2} \dot{S} v=-\int_{\Omega} a(u) \mathcal{A} \nabla S(u) \cdot \nabla v-\int_{\Omega} b(u) S(u)^{3} v d i v V+ \\
\int_{\Omega} \operatorname{div}(f V) v \quad \forall v \in H^{1}(\Omega),
\end{gathered}
$$


being $\mathcal{A}=\operatorname{div} V-\left(D V+D V^{T}\right)$.

Proof. We start by deriving the formula of the material derivative of the solution map. Define $S_{0}=S(u)$ and $S_{t}: \Omega \rightarrow \mathbb{R}, S_{t}=S\left(u_{t}\right) \circ \phi_{t}$. Then, applying the change of variables induced by the map $\phi_{t}$, it holds that

$$
\int_{\Omega} a(u) A(t) \nabla S_{t} \cdot \nabla v+\int_{\Omega} b(u) S_{t}^{3} v\left|\operatorname{det} D \phi_{t}\right|=\int_{\Omega}\left(f \cdot \phi_{t}\right) v\left|\operatorname{det} D \phi_{t}\right| \quad \forall v \in H^{1}(\Omega),
$$

where $A(t)=D \phi_{t}^{-T} D \phi_{t}^{-1}\left|\operatorname{det} D \phi_{t}\right|$. By computation,

$$
\frac{d}{d t} A(t)=\mathcal{A}=(\operatorname{div} V) I-\left(D V^{t}+D V\right) \quad \text { and } \quad \frac{d}{d t}\left|\operatorname{det} D \phi_{t}\right|=\operatorname{div} V .
$$

Subtract (2.3) from (5.7) and divide by $t$ : then $w_{t}=\frac{S_{t}-S_{0}}{t}$ is the solution of

$$
\begin{gathered}
\int_{\Omega} a(u) A(t) \nabla w_{t} \cdot \nabla v+\int_{\Omega} b(u) q_{t} w_{t} v\left|\operatorname{det}\left(D \phi_{t}\right)\right|=-\int_{\Omega} a(u) \frac{A(t)-I}{t} \nabla S_{0} \cdot \nabla v \\
-\int_{\Omega} \frac{\left|\operatorname{det}\left(D \phi_{t}\right)\right|-1}{t} b(u) S_{0}^{3} v+\int_{\Omega} \frac{1}{t}\left(f \circ \phi_{t}\right) v\left|\operatorname{det}\left(D \phi_{t}\right)\right|-\int_{\Omega} \frac{1}{t} f v
\end{gathered}
$$

$\forall v \in H^{1}(\Omega)$, where the norm of the right-hand side in the dual space of $H^{1}(\Omega)$ is bounded by

$$
\begin{aligned}
& \left\|\frac{A-I}{t}\right\|_{L^{\infty}(\Omega)}\left\|S_{0}\right\|_{H^{1}(\Omega)}+\left\|\frac{\left|\operatorname{det}\left(D \phi_{t}\right)\right|-1}{t}\right\|_{L^{\infty}(\Omega)}\left\|S_{0}\right\|_{H^{1}(\Omega)} \\
& \quad+\left\|\frac{\left|\operatorname{det}\left(D \phi_{t}\right)\right|-1}{t}\right\|_{L^{\infty}(\Omega)}\|f\|_{L^{2}(\Omega)}+C\left(\|V\|_{C(\Omega)}\right)\|f\|_{H^{1}(\Omega)} \leq C_{F},
\end{aligned}
$$

being $C_{F}$ independent of $t$. Moreover, the matrix $A(t)$ is symmetric positive definite: $(A(t) y) \cdot y \geq \frac{1}{2}\|y\|^{2} \forall y \in \mathbb{R}^{2}, \forall t$. Together with the property that $q_{t}=u_{t}^{2}+u_{t} u+u^{2} \geq \frac{3}{4} u^{2}$, and thanks to Proposition 2.4 and to the Poincaré inequality in Lemma 2.1,

$$
\left\|w_{t}\right\|_{H^{1}}^{2} \leq C\left(k\left\|\nabla w_{t}\right\|_{L^{2}}^{2}+\frac{3}{4} m^{2 / 3}\left\|w_{t}\right\|_{L^{2}\left(\Omega^{d_{0}}\right)}^{2}\right) \leq C_{F}\left\|w_{t}\right\|_{H^{1}} .
$$

Thus, $\left\|w_{t}\right\|_{H^{1}}$ is bounded independently of $t$, from which it follows that $\left\|S_{t}-S_{0}\right\|_{H^{1}(\Omega)} \leq$ $C t$ and that every sequence $\left\{w_{n}\right\}=\left\{w_{t_{n}}, t_{n} \rightarrow 0\right\}$ is bounded in $H^{1}(\Omega)$, thus $w_{t} \stackrel{H^{1}}{\longrightarrow} w \in$ $H^{1}(\Omega)$. We aim at proving that $w$ is also the limit of $w_{t}$ in the strong convergence, which entails that

$$
\dot{S}(u)[V]:=\lim _{t \rightarrow 0} \frac{S_{t}-S_{0}}{t}=w .
$$

First of all, we show that $w$ is the solution of problem (5.6). It follows from (5.8), since $q_{t} w_{t}=\frac{1}{t}\left(S_{t}^{3}-S_{0}^{3}\right)=\frac{1}{t}\left(\left(S_{0}+t w_{t}\right)^{3}-S_{0}^{3}\right)=3 S_{0}^{2} w_{t}+3 t S_{0} w_{t}^{2}+t^{2} w_{t}^{3}$, that

$$
\begin{gathered}
\int_{\Omega} a(u) A(t) \nabla w_{t} \cdot \nabla v+\int_{\Omega} b(u) 3 S_{0}^{2} w_{t} v\left|\operatorname{det} D \phi_{t}\right|=-\int_{\Omega} a(u) \frac{A(t)-I}{t} \nabla S_{0} \cdot \nabla v \\
-\int_{\Omega} \frac{\left|\operatorname{det} D \phi_{t}\right|-1}{t} b(u) S_{0}^{3} v-\int_{\Omega} b(u) 3 t S_{0} w_{t}^{2} v\left|\operatorname{det} D \phi_{t}\right|-\int_{\Omega} b(u) t^{2} w_{t}^{3} v\left|\operatorname{det} D \phi_{t}\right| \\
\quad+\int_{\Omega}\left(f \circ \phi_{t}\right) \frac{\left|\operatorname{det} D \phi_{t}\right|-1}{t} v-\int_{\Omega} \frac{\left(f \circ \phi_{t}\right)-f}{t} v \quad \forall v \in H^{1}(\Omega) .
\end{gathered}
$$


Taking the limit as $t \rightarrow 0$ and by the weak convergence of $w_{t}$ in $H^{1}$, we recover the same expression as in (5.6). One may eventually show that $w_{t} \stackrel{H^{1}}{\longrightarrow} w$. In order to do this we start proving that

$$
\int_{\Omega} a(u) A(t)\left|\nabla w_{t}\right|^{2}+\int_{\Omega} b(u)\left|\operatorname{det} D \phi_{t}\right| 3 S_{0}^{2} w_{t}^{2} \rightarrow \int_{\Omega} a(u)|\nabla w|^{2}+\int_{\Omega} b(u) 3 S_{0}^{2} w^{2} .
$$

Indeed, take (5.9) and substitute $v=w_{t}$ : using the weak convergence of $w_{t}$ in the righthand side, we obtain that

$$
\begin{aligned}
\int_{\Omega} a(u) A(t)\left|\nabla w_{t}\right|^{2} & +\int_{\Omega} b(u)\left|\operatorname{det} D \phi_{t}\right| 3 S_{0}^{2} w_{t}^{2} \rightarrow-\int_{\Omega} a(u) \mathcal{A} \nabla S_{0} \cdot \nabla w-\int_{\Omega} \operatorname{div} V b(u) S_{0}^{3} w \\
& +\int_{\Omega} f w \operatorname{div} V-\int_{\Omega} \nabla f \cdot V w \stackrel{(5.6)}{=} \int_{\Omega} a(u)|\nabla w|^{2}+\int_{\Omega} b(u) 3 S_{0}^{2} w^{2} .
\end{aligned}
$$

We then compute:

$$
\begin{aligned}
& \int_{\Omega} a(u) A(t)\left|\nabla\left(w_{t}-w\right)\right|^{2}+\int_{\Omega} b(u) 3 S_{0}^{2}\left(w_{t}-w\right)^{2}\left|\operatorname{det} D \phi_{t}\right|= \\
& \int_{\Omega} a(u) A(t)\left|\nabla w_{t}\right|^{2}+\int_{\Omega} a(u) A(t)|\nabla w|^{2}-2 \int_{\Omega} a(u) A(t) \nabla w_{t} \cdot \nabla w \\
& +\int_{\Omega} b(u) 3 S_{0}^{2} w_{t}^{2}\left|\operatorname{det} D \phi_{t}\right|+\int_{\Omega} b(u) 3 S_{0}^{2} w^{2}\left|\operatorname{det} D \phi_{t}\right|-2 \int_{\Omega} b(u) 3 S_{0}^{2} w_{t} w\left|\operatorname{det} D \phi_{t}\right| .
\end{aligned}
$$

Using (5.10), the convergence of $A$ to $I$ and of $\left|\operatorname{det} D \phi_{t}\right|$ to 1 , and the fact that $w_{t} \stackrel{H^{1}}{\longrightarrow} w$, we derive that

$$
\int_{\Omega} a(u)\left|\nabla\left(w_{t}-w\right)\right|^{2}+\int_{\Omega} b(u) 3 S_{0}^{2}\left(w_{t}-w\right)^{2} \rightarrow 0
$$

A combination of the Proposition 2.4 and of the Poincaré inequality in Lemma 2.1 allows to conclude that also $\left\|w_{t}-w\right\|_{H^{1}} \rightarrow 0$.

We now prove the necessary optimality conditions for the optimization problem (5.1). The derivative of the quadratic part of the cost functional $J$ can be easily computed by means of the material derivative of the solution map:

$$
\begin{aligned}
& \lim _{t \rightarrow 0} \frac{1}{2} \int_{\partial \Omega} \frac{\left(S\left(u_{t}\right)-y_{\text {meas }}\right)^{2}\left|\operatorname{det}\left(D \phi_{t}\right)\right|-\left(S_{0}-y_{\text {meas }}\right)^{2}}{t} \quad\left(\text { since } S\left(u_{t}\right)=S_{t} \text { on } \partial \Omega\right) \\
& =\lim _{t \rightarrow 0} \frac{1}{2} \int_{\partial \Omega}\left(S_{t}-y_{\text {meas }}\right)^{2} \frac{\left|\operatorname{det}\left(D \phi_{t}\right)\right|-1}{t}+\lim _{t \rightarrow 0} \frac{1}{2} \int_{\partial \Omega} \frac{\left(S_{t}-y_{\text {meas }}\right)^{2}-\left(S_{0}-y_{\text {meas }}\right)^{2}}{t} \\
& =\frac{1}{2} \int_{\partial \Omega}\left(S_{0}-y_{\text {meas }}\right)^{2} \operatorname{div} V+\int_{\partial \Omega} \dot{S}(u)[V]\left(S_{0}-y_{\text {meas }}\right),
\end{aligned}
$$

and the first integral in the latter expression vanishes since $V=0$ on $\Omega_{d_{0}}$. On the other hand, using Lemma 10.1 of [27] and the remark 10.2, we recover the expression for the derivative of the Total Variation of $u$, which is the same reported in (5.5).

The optimality conditions reported in (5.4) are, at the best of our knowledge, the most general result which can be obtained in this case, i.e. by simply assuming that 
$u=\chi_{\omega}$ and $\omega$ is a set of finite perimeter. We point out that, assuming more a priori information on $u$, it is possible to recover from (5.5) the expression of the shape derivative of the cost functional $J$. The following proposition can be rigorously proved by means of an argument similar as the one used in [2], except for the derivative of the perimeter penalization, which can be found in Section 9.4.3 in [21].

Proposition 5.2. Suppose that $\omega \subset \Omega$ is open, connected, well separated from the boundary $\partial \Omega$ and regular (at least of class $C^{2}$ ), and $u=\chi_{\omega}$. Then, the expression of the shape derivative of the cost functional $J$ along a smooth vector field $V$ is:

$$
D J(u)[V]=\int_{\partial \omega}\left[(1-k)\left(\nabla_{\tau} S(u) \cdot \nabla_{\tau} w+\frac{1}{k} \nabla_{\nu} S(u)^{e} \cdot \nabla_{\nu} w^{e}\right)+S(u)^{3} w+\mathcal{H}\right] V \cdot \nu,
$$

where $w$ is the solution of the adjoint problem (see (2.15)). The gradients $\nabla S(u)$ and $\nabla w$ are decomposed in the normal and tangential component with respect to the boundary $\partial \omega$, and due to the transmission condition of the direct problem their normal components are discontinuos across $\partial \omega$ : the valued assumed in $\Omega \backslash \omega$ is marked as $\nabla_{\nu} S(u)^{e}$. The term $\mathcal{H}$ is instead the curvature of the boundary.

For the sake of completeness, we point out that the latter result can be easily generalized to the case in which $\omega$ is the union of $N_{c}$ disjoint, well separated, components, each of them satisfying the expressed hypotheses. Thanks to the results recently obtained in [9], we expect formula (5.13) to be valid also under milder assumption, in particular for polygons.

We aim at demonstrating that the expression of the shape derivative reported in (5.4) is the limit, as $\varepsilon \rightarrow 0$, of the shape derivative of the relaxed cost functional $J_{\varepsilon}$ (defined as in (5.3), replacing $u$ by $u_{\varepsilon}$ and $J$ by $J_{\varepsilon}$ ). In order to accomplish this result, we need to introduce necessary optimality conditions for the relaxed problem (2.12) which are different from the ones reported in Proposition 2.10 and can be derived by the same technique as in Proposition 5.1 as shown in the following result.

Proposition 5.3. If $u_{\varepsilon}$ is a solution of (2.12), then

$$
D J_{\varepsilon}\left(u_{\varepsilon}\right)[V]=0 \quad \text { for all the smooth vector fields } V,
$$

The expression of the derivative is given by:

$$
\begin{aligned}
D J_{\varepsilon}\left(u_{\varepsilon}\right)[V]= & \int_{\partial \Omega}\left(S\left(u_{\varepsilon}\right)-y_{\text {meas }}\right) \dot{S}\left(u_{\varepsilon}\right)[V]+\alpha \varepsilon \int_{\Omega}\left|\nabla u_{\varepsilon}\right|^{2} \operatorname{div} V \\
& -2 \alpha \varepsilon \int_{\Omega} D V \nabla u_{\varepsilon} \cdot \nabla u_{\varepsilon}+\frac{\alpha}{\varepsilon} \int_{\Omega} u_{\varepsilon}\left(1-u_{\varepsilon}\right) \operatorname{div} V
\end{aligned}
$$

where $\dot{S}\left(u_{\varepsilon}\right)[V]$ solves the same problem as in (5.6), replacing $u$ with $u_{\varepsilon}$.

Proof. The same strategy as in the proof of Proposition 5.1 can be adapted to compute $\dot{S}\left(u_{\varepsilon}\right)[V]$ and the derivative of the first term of the cost functional. We now derive with the same computational rules the relaxed penalization term. Recall

$$
F_{\varepsilon}\left(u_{\varepsilon}\right)=\alpha \varepsilon \int_{\Omega}\left|\nabla u_{\varepsilon}\right|^{2}+\frac{\alpha}{\varepsilon} \int_{\Omega} \psi\left(u_{\varepsilon}\right),
$$


being $\psi: \mathbb{R} \rightarrow \mathbb{R}, \psi(x)=x(1-x)$. After the deformation from $u_{\varepsilon}$ to $u_{\varepsilon} \circ \phi_{t}^{-1}$ and applying the change of variables induced by $\phi_{t}$,

$$
F_{\varepsilon}\left(u_{\varepsilon} \circ \phi_{t}^{-1}\right)=\alpha \varepsilon \int_{\Omega} A(t) \nabla u_{\varepsilon} \cdot \nabla u_{\varepsilon}+\frac{\alpha}{\varepsilon} \int_{\Omega} \psi \circ u_{\varepsilon} \circ \phi_{t}^{-1}
$$

Hence,

$$
\begin{aligned}
\dot{F}_{\varepsilon}\left(u_{\varepsilon}\right)[V] & =\lim _{t \rightarrow 0} \frac{F_{\varepsilon}\left(u_{\varepsilon} \circ \phi_{t}^{-1}\right)-F_{\varepsilon}\left(u_{\varepsilon}\right)}{t}=\alpha \varepsilon \int_{\Omega} \mathcal{A} \nabla u_{\varepsilon} \cdot \nabla u_{\varepsilon}+\alpha \varepsilon \frac{\alpha}{\varepsilon} \int_{\Omega} \psi\left(u_{\varepsilon}\right) \operatorname{div} V= \\
& =\alpha \varepsilon \int_{\Omega}\left|\nabla u_{\varepsilon}\right|^{2} d i v V-\alpha \varepsilon \int_{\Omega}\left(D V+D V^{T}\right) \nabla u_{\varepsilon} \cdot \nabla u_{\varepsilon}+\frac{\alpha}{\varepsilon} \int_{\Omega} u_{\varepsilon}\left(1-u_{\varepsilon}\right) \operatorname{div} V
\end{aligned}
$$

which is the same expression as in (5.15), since $D V^{T} \nabla v \cdot \nabla v=D V \nabla v \cdot \nabla v$.

We point out that the optimality conditions deduced in the latter proposition are not equivalent to the ones expressed in Proposition 2.10 via the Fréchet derivative of $J_{\varepsilon}$. Nevertheless, if $u_{\varepsilon}$ satisfies (2.13)-(2.14), then it also satisfies (5.14) (it is sufficient to consider in (2.13) $v=u_{\varepsilon} \circ \phi_{t}^{-1}$, which belongs to $\mathcal{K}$ thanks to the regularity of $V$ ), whereas the contrary is not valid in general. In particular, due to the regularity of the perturbation fields $V$, the optimality conditions (5.14) do not take into account possible topological changes of the inclusion: for example, the number of connected components of $\omega$ cannot change. We remark that this holds also for the optimality conditions (5.4) for the sharp problem, and consists in a limitation for the effectiveness of the reconstruction via a shape derivative approach: the initial guess of the reconstruction algorithm and the exact inclusion must be diffeomorphic.

We are now able to show the sharp interface limit of the expression of the shape derivative of the relaxed cost functional $J_{\varepsilon}$ as $\varepsilon \rightarrow 0$, which is done in the following proposition.

Proposition 5.4. Consider a family $\bar{u}_{\varepsilon}$ s.t. $\bar{u}_{\varepsilon} \in \mathcal{K} \forall \varepsilon>0$ and $\bar{u}_{\varepsilon} \stackrel{L^{1}}{\rightarrow} \bar{u} \in B V(\Omega)$ as $\varepsilon \rightarrow 0$. Then,

$$
D J_{\varepsilon}\left(\bar{u}_{\varepsilon}\right)[V] \rightarrow D J(\bar{u})[V] \quad \text { for every smooth vector field } V .
$$

Proof. We follow a similar argument as in the proof of [12, Theorem 21]. Thanks to Proposition 2.5, $\bar{u}_{\varepsilon} \stackrel{L^{1}}{\longrightarrow} \bar{u} \Rightarrow S\left(\bar{u}_{\varepsilon}\right) \stackrel{H^{1}}{\longrightarrow} S(\bar{u})$. Also $\dot{S}\left(\overline{u_{\varepsilon}}\right)[V] \stackrel{H^{1}}{\longrightarrow} \dot{S}(\bar{u})[V]:$ the proof is done by subtracting the equations of which $\dot{S}\left(\bar{u}_{\varepsilon}\right)[V]$ and $\dot{S}(\bar{u})[V]$ and verifying that the norm of their difference is controlled by the norm of $S\left(\bar{u}_{\varepsilon}\right)-S(\bar{u})$ in $H^{1}(\Omega)$. Thanks to these results, surely

$$
\int_{\Omega}\left(S\left(u_{\varepsilon}\right)-y_{\text {meas }}\right) \dot{S}\left(\bar{u}_{\varepsilon}\right)[V] \rightarrow \int_{\Omega}\left(S(u)-y_{\text {meas }}\right) \dot{S}(\bar{u})[V] .
$$

Eventually, the convergence

$$
\alpha \varepsilon \int_{\Omega}\left|\nabla \overline{u_{\varepsilon}}\right|^{2} \operatorname{div} V-2 \alpha \varepsilon \int_{\Omega} D V \nabla \overline{u_{\varepsilon}} \cdot \nabla \overline{u_{\varepsilon}}+\frac{\alpha}{\varepsilon} \int_{\Omega} \overline{u_{\varepsilon}}\left(1-\overline{u_{\varepsilon}}\right) \operatorname{div} V \rightarrow \int_{\Omega}(\operatorname{div} V-D V \nu \cdot \nu) d|D \bar{u}|
$$


is proved in [25], Theorem 4.2 (see also annotations in [12], proof of Theorem 21). In particular, we point out that this implies, together with Proposition 2.9, that the expression of the optimality condition for the phase field problem converges, as $\varepsilon \rightarrow 0$, to the one in the sharp case.

5.2. Comparison with the shape derivative algorithm In this section, we report some results of the application of the algorithm based on the shape derivative. In the implementation, we take advantage of the Finite Element method to solve the direct and adjoint problems and compute the shape gradient as in (5.13). We consider an initial guess for the inclusion (in all the simulations reported, the initial guess is a disc centered in the origin with radius 0.02 ) and discretize its boundary with a finite number of points, which always coincide with vertices of the numerical mesh. We iteratively perturb the inclusion by moving the boundary with a normal vector field $V$ which is the projection in the Finite Element space of the shape gradient reported in (5.13) (see e.g. [22] for more details). After the descent direction is determined, a backtracking scheme is implemented (see [36]), in order to guarantee the decrease of the cost functional $J$ at each iteration. As in the case of Algorithm 1, we start from the initial guess $u^{0} \equiv 0$ and take advantage of $N_{f}=2$ measurements, associated to the same source terms. The main parameters of this set of simulations are reported in Table 5.1.

\begin{tabular}{c|c|c}
$\alpha$ & maxstep & tol \\
\hline $10^{-3}$ & 10 & $10^{-6}$
\end{tabular}

Table 5.1: Values of the main parameters

In Figure 5.1 we report the results of the reconstruction with the shape gradient algorithm compared to the ones of the Parabolic Obstacle problem (with $\varepsilon=\frac{1}{16 \pi}$ and with mesh adaptation). Each result is endowed with a plot of the evolution of the cost functional throughout time (in particular, of $J_{P D E}(u)=\frac{1}{2}\left\|S(u)-y_{\text {meas }}\right\|_{L^{2}(\partial \Omega)}$ ). The reconstruction achieved by the shape gradient algorithm is qualitatively as accurate as the phase-field one. The first method is less expensive in terms of number of iterations. Nevertheless, it requires a priori knowledge about the topology of the unknown inclusion.

Acknowledgments. The authors are grateful to the reviewers for their careful reading and insightful comments on the paper. E. Beretta and M. Verani thank the New York University in Abu Dhabi for its kind hospitality that permitted a further development of the present research. We acknowledge the use of the MATLAB library redbKIT [35] for the numerical simulations presented in this work.

\section{References.}




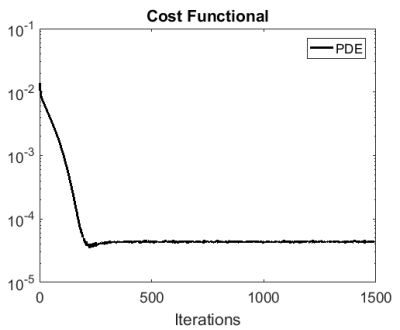

(a) Shape gradient, evolution of the cost functional

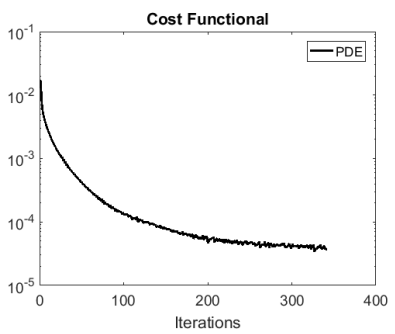

(d) Shape gradient, evolution of the cost functional

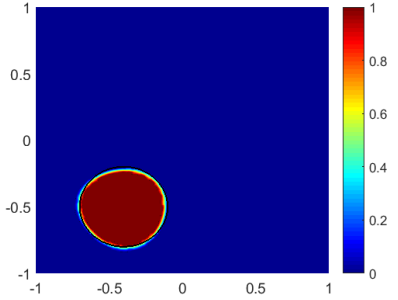

(b) Shape gradient, $N_{\text {tot }}=1494$

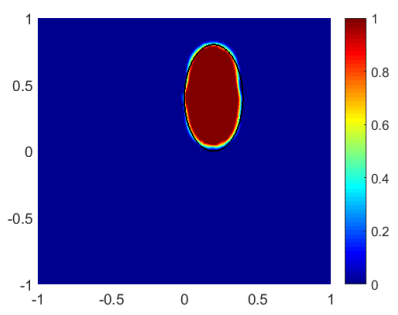

(e) Shape gradient, $N_{t o t}=301$

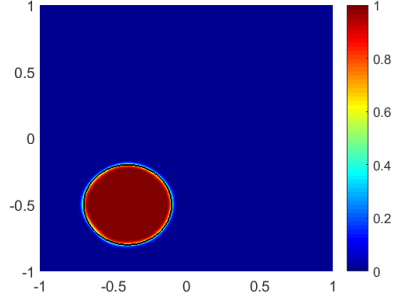

(c) Phase field, $\varepsilon=\frac{1}{16 \pi}, N_{\text {tot }}=1869$

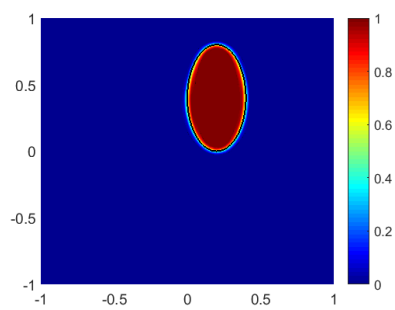

(f) Phase field, $\varepsilon=\frac{1}{16 \pi}, N_{t o t}=1503$

Figure 5.1: Shape gradient algorithm: result comparison

[1] L. Afraites, M. Dambrine, and D. Kateb. "Conformal mappings and shape derivatives for the transmission problem with a single measurement". In: Numer. Func. Anal. Opt. 28 (2007), pp. 519-551.

[2] H. Ammari, E. Beretta, E. Francini, H. Kang, and M. Lim. "Optimization algorithm for reconstructing interface changes of a conductivity inclusion from modal measurements". In: Math. Comp. 79(271) (2010), pp. 1757-1777.

[3] H. Ammari, J. Garnier, V. Jugnon, and H. Kang. "Stability and resolution analysis for a topological derivative based imaging functional". In: SIAM Journal on Control and Optimization 50.1 (2012), pp. 48-76.

[4] H. Ammari and H. Kang. Reconstruction of small inhomogeneities from boundary measurements. Lectures Notes in Mathematics Series, Volume 1846. Springer, 2004.

[5] M. Bachmayr and M. Burger. "Iterative total variation schemes for nonlinear inverse problems". In: Inverse Problems 25.10 (2009), p. 105004.

[6] S. Baldo. "Minimal interface criterion for phase transitions in mixtures of CahnHilliard fluids". In: Ann. IHP Anal. Non Linéaire. Vol. 7. 2. 1990, pp. 67-90.

[7] E. Beretta, C. Cavaterra, M.C. Cerutti, A. Manzoni, and L. Ratti. "An inverse problem for a semilinear parabolic equation arising from cardiac electrophysiology". In: Inverse Problems (2017). 
[8] E. Beretta, M.C. Cerutti, A. Manzoni, and D. Pierotti. "An asymptotic formula for boundary potential perturbations in a semilinear elliptic equation related to cardiac electrophysiology". In: Math. Mod. and Meth. in Appl. S. 26(04) (2016), pp. $645-670$.

[9] E. Beretta, E. Francini, and S. Vessella. "Differentiability of the Dirichlet to Neumann Map Under Movements of Polygonal Inclusions with an Application to Shape Optimization". In: SIAM Journal on Mathematical Analysis 49.2 (2017), pp. $756-776$.

[10] E. Beretta, A. Manzoni, and L. Ratti. "A reconstruction algorithm based on topological gradient for an inverse problem related to a semilinear elliptic boundary value problem". In: Inverse Problems 33.3 (2017).

[11] M. Bergounioux and K. Kunisch. "Augemented Lagrangian Techniques for Elliptic State Constrained Optimal Control Problems". In: SIAM Journal on Control and Optimization 35.5 (1997), pp. 1524-1543.

[12] L. Blank, H. Garcke, C. Hecht, and C. Rupprecht. "Sharp interface limit for a phase field model in structural optimization". In: SIAM J. Control Optim. 54.3 (2016), pp. 1558-1584.

[13] M. Burger. "Levenberg-Marquardt level set methods for inverse obstacle problems". In: Inverse problems 20.1 (2003), p. 259.

[14] A. Carpio and M. L. Rapún. "Topological derivatives for shape reconstruction". In: Inverse problems and imaging. Springer, 2008, pp. 85-133.

[15] T. F. Chan and X. Tai. "Level set and total variation regularization for elliptic inverse problems with discontinuous coefficients". In: J. Comput. Phys. 193(1) (2004), pp. 40-66.

[16] C. E. Chávez, N. Zemzemi, Y. Coudière, F. Alonso-Atienza, and D. Alvarez. "Inverse problem of electrocardiography: Estimating the location of cardiac ischemia in a 3d realistic geometry". In: International Conference on Functional Imaging and Modeling of the Heart. Springer. 2015, pp. 393-401.

[17] Z. Chen and J. Zou. "An augmented Lagrangian method for identifying discontinuous parameters in elliptic systems". In: SIAM Journal on Control and Optimization 37.3 (1999), pp. 892-910.

[18] P. G. Ciarlet, M. H. Schultz, and R.S. Varga. "Numerical methods of high-order accuracy for nonlinear boundary value problems". In: Numerische Mathematik 9.5 (1967), pp. 394-430.

[19] P. Colli Franzone, L.F. Pavarino, and S. Scacchi. Mathematical Cardiac Electrophysiology. Vol. 13. MS\&A. Springer, 2014.

[20] K. Deckelnick, C. M Elliott, and V. Styles. "Double obstacle phase field approach to an inverse problem for a discontinuous diffusion coefficient". In: Inverse Problems 32 (2016).

[21] M. C Delfour and J.-P. Zolésio. Shapes and geometries: metrics, analysis, differential calculus, and optimization. SIAM, 2011. 
[22] G. Dogan, P. Morin, R. H. Nochetto, and M. Verani. "Discrete gradient flows for shape optimization and applications". In: Computer methods in applied mechanics and engineering 196.37 (2007), pp. 3898-3914.

[23] H.W. Engl, M. Hanke, and A. Neubauer. Regularization of Inverse Problems. Mathematics and Its Applications. Springer Netherlands, 1996. ISBN: 9780792341574.

[24] L.C. Evans and R.F. Gariepy. Measure theory and fine properties of functions. CRC press, 2015.

[25] H. Garcke. "The $\Gamma$-limit of the Ginzburg-Landau energy in an elastic medium". In: $A M S A 18.2$ (2008), pp. 345-379.

[26] D. Gilbarg and N. S. Trudinger. Elliptic Partial Differential Equations of Second Order. Grundlehren der mathematischen Wissenschaften. Springer, 1998.

[27] E. Giusti. Minimal surfaces and functions of bounded variation. Springer, 1984.

[28] F. Hettlich and W. Rundell. "The determination of a discontinuity in a conductivity from a single boundary measurement". In: Inverse Problems 14 (1998), 311-318.

[29] M. Hintermüller and A. Laurain. "Electrical impedance tomography: from topology to shape". In: Control \& Cybernetics 37.4 (2008).

[30] Victor Isakov. "On uniqueness of recovery of a discontinuous conductivity coefficient". In: Communications on pure and applied mathematics 41(7) (1988), pp. $865-877$.

[31] R. V. Kohn and M. Vogelius. "Relaxation of a variational method for impedance computed tomography". In: Communications on Pure and Applied Mathematics 40.6 (1987), pp. 745-777.

[32] V. Kolehmainen, S. R. Arridge, W. R. B. Lionheart, M. Vauhkonen, and J. P. Kaipio. "Recovery of region boundaries of piecewise constant coefficients of an elliptic PDE from boundary data". In: Inverse Problems 15.5 (1999), p. 1375.

[33] E. H. Lieb and M. Loss. "Analysis-second edition, Graduate Studies in Mathematics". In: American Mathematical Society 14 (2001).

[34] M. Lysaker, B. F. Nielsen, and A. Tveito. "On the use of the resting potential and level set methods for identifying ischemic heart disease: An inverse problem". In: J. Comput. Phys. 220 (2007), pp. 772-790.

[35] F. Negri. redbKIT Version 2.2. http:/redbkit.github.io/redbKIT/. Copyright (c) 2015-2017, Ecole Polytechnique Fédérale de Lausanne (EPFL) All rights reserved. 2016.

[36] J. Nocedal and S. J. Wright. Numerical Optimization. 2nd. Springer, 2006.

[37] L. Rondi and F. Santosa. "Enhanced electrical impedance tomography via the Mumford-Shah functional". In: ESAIM: Control, Optimisation and Calculus of Variations 6 (2001), pp. 517-538.

[38] F. Santosa. "A level-set approach for inverse problems involving obstacles". In: ESAIM Control Optim. Calc. Var. 1 (1996), pp. 17-33. 
[39] J. Sundnes, G. T. Lines, X. Cai, B. F. Nielsen, K.A. Mardal, and A. Tveito. Computing the electrical activity in the heart. Monographs in Computational Science and Engineering Series, Volume 1. Springer, 2006.

[40] L. Tung. A bidomain model for describing ischemic myocardial D-C potentials. Ph.D. Thesis. MIT, Cambridge, MA, 1978. 